\title{
Hyperthermostable Binding Molecules on Phage: Assay Components for Point-of-Care Diagnostics for Active Tuberculosis Infection
}

\author{
Ning Zhao ${ }^{\mathrm{a}}$, John Spencer ${ }^{\mathrm{b}}$, Margaret A. Schmitt ${ }^{\mathrm{a}}$, John D. Fisk ${ }^{\mathrm{a}, \mathrm{c}, \mathrm{d} *}$
}

Keywords: Mycobacterium tuberculosis, ornithine transcarbamylase, phage display, alternative scaffold, molecular recognition, diagnostics

Abbreviations: TB, tuberculosis; Mtb, Mycobacterium tuberculosis; Mtb OTC, Mycobacterium tuberculosis ornithine transcarbamylase; CV, column volumes; HRP, horseradish peroxidase; TMB, tetramethylbenzidine; PBST, PBS with $0.05 \%$ Tween 20 (v/v); amp, ampicillin; carb, carbenicillin; cam, chloramphenicol; kan, kanamycin; tet, tetracycline; DSC, differential scanning calorimetry; LAM, liparabinomannan 


\begin{abstract}
Tuberculosis is the leading cause of death from infectious disease worldwide. The low sensitivity, extended processing time, and high expense of current diagnostics are major challenges to the detection and treatment of tuberculosis. Mycobacterium tuberculosis ornithine transcarbamylase (Mtb OTC, Rv1656) has been identified in the urine of patients with active TB infection and is a promising target for point-of-care diagnostics. Specific binding proteins with low nanomolar affinities for Mtb OTC were selected from a phage display library built upon a hyperthermostable Sso7d scaffold. Phage particles displaying Sso7d variants were utilized to generate a sandwich ELISA-based assay for $M t b$ OTC. The assay response is linear between 2 $\mathrm{ng} / \mathrm{mL}$ and $125 \mathrm{ng} / \mathrm{mL}$ recombinant $\mathrm{Mtb}$ OTC and has a limit of detection of $400 \mathrm{pg} / \mathrm{mL}$ recombinant $M t b$ OTC. The assay employing a phage-based detection reagent is comparable to commercially-available antibody-based biosensors. Importantly, the assay maintains functionality at both neutral and basic $\mathrm{pH}$ in presence of salt and urea over the range of concentrations typical for human urine. Phage-based diagnostic systems may feature improved physical stability and cost of production relative to traditional antibody-based reagents, without sacrificing specificity and sensitivity.
\end{abstract}




\section{Introduction}

Tuberculosis (TB) has surpassed HIV as the leading cause of death from infectious disease worldwide [1]. TB, caused by the bacillus Mycobacterium tuberculosis (Mtb), typically affects the lungs, but can also affect other organs or tissues. Nearly $1 / 3$ of the world's population is chronically infected with TB (latent TB), but only a small percentage of those infected develop disease. In 2014, 9.6 million new cases of active TB and 1.2 million TB deaths were recorded [2]. The high mortality of TB is due primarily to the difficulty of diagnosis in resource-limited areas rather than a difficulty in treatment. The detection rate for TB worldwide averages only $63 \%$, and as few as $50 \%$ of the expected cases in Africa are diagnosed [3]. In order to combat the global TB epidemic, fast, sensitive and affordable diagnostics of active TB infection are in high demand [4]. It has been estimated that even moderately accurate point-of-care diagnostics for TB could reduce the annual mortality by $30 \%-50 \%$ [5]. The current diagnostic standard for TB is a culture-based screen that requires laboratory facilities and weeks of culture time for results, making it unsuitable as a point-of-care diagnostic. The most common front line TB diagnostic is sputum smear microscopy, which suffers from low sensitivity and also requires minimal laboratory facilities.

Diagnostic methods directly targeting TB antigens are of particular interest because direct antigen detection assays have the potential to discriminate between latent and active TB [6, 7]. Differentiating active infection from latent exposure is a critical necessity, as only active infection requires treatment. The high exposure rate $(\sim 1 / 3$ of the world's population) has rendered traditional immunological tests useless for diagnosis. The ideal diagnostic will rapidly detect active TB in patients through direct detection of $M t b$ antigens and employ bodily secretions that limit the health risk to medical providers. Blood is problematic because of the high prevalence of HIV in TB endemic areas; sputum is problematic because secretions can contain infectious bacteria. Diagnosis using urine is of interest because of the ease of sample collection and relative safety compared to sputum sampling [8].

Among all urine antigen candidates, lipoarabinomannan (LAM), which is a major component of the $M t b$ bacterial cell wall, is the most frequently targeted [9, 10]. Several clinical studies have screened individuals with suspected TB by anti-LAM ELISA. Diagnostic field tests in Ethiopia showed sensitivity of $74.0 \%$ and specificity of $86.9 \%$ and in Tanzania a sensitivity of $80.3 \%$ and specificity of $99 \%$ [11]. Sensitivity describes the ability to detect, and specificity describes the accuracy of detection, the ratio of true to false determinations. Sensitivity and specificity for TB diagnostics are measured in relation to culture-based screening. Another 4 studies on LAM ELISA screens showed consistently poor overall performance with sensitivities ranging from $13 \%$ to $51 \%$ [12-15]. The combined studies suggest little consistency for the detection of urine LAM in patients with suspected TB [8]. Antibody tests in general, and specifically those that target glycans such as LAM, are problematic in point-of-care settings. Antibody molecules are difficult and expensive to synthesize and have poor stability. Furthermore, saccharide-targeting antibodies generally have low affinities relative to protein or peptide recognizing species, reducing the sensitivity of diagnostic tests [16].

The effort to identify new diagnostic molecular markers of TB in various bodily fluids is ongoing $[10,17-20]$. To improve TB diagnosis sensitivity and reliability, tests for multiple molecular markers of disease need to be developed. The $M t b$ protein ornithine transcarbamylase 
(Mtb OTC, Rv1656) has been identified in active TB patients' urine by mass spectrometry [21]. Antibody-based sandwich ELISA tests employing Mtb OTC polyclonal antibodies were used to evaluate the urine of 16 pulmonary TB patients and 16 healthy patients. The urine ELISA was able to identify the presence of Mtb OTC in 6 out of 16 pulmonary TB patients and none of the 16 healthy control subjects [21].

Ideally, the stability, cost of production and reliability of the molecular components of diagnostics need to be improved to allow their use in point-of-care settings [22]. We describe the selection of tight, specific binding molecules against Mtb OTC from an alternative scaffold protein library by phage display [23]. An alternative scaffold is a protein other than an antibody that is diversified and selected in vitro to bind a specific target. Alternative scaffolds are increasingly being employed to select binding molecules for diverse applications where the cost or physical properties of antibodies are prohibitive [24, 25]. The alternative scaffold utilized for the selection of $M t b$ OTC binding molecules is derived from a small, cysteine-free DNA-binding protein, Sso7d, from the hyperthermophilic archeon Sulfolobus sulfataricus. Sso7d is a representative of an abundant class of 6-8 $\mathrm{kDa}$, putative histone-like DNA-binding proteins isolated from thermophilic Sulfolobus species [26]. Sso7d and the closely-related Sac7d from Sulfolobus acidocaldarius are $7 \mathrm{kDa}, 63$ amino acid proteins with identical three-dimensional structures that share $85 \%$ sequence identity. Sso7d and Sac7d have been extensively studied as model thermophilic and DNA-binding proteins, with melting temperatures of nearly $100{ }^{\circ} \mathrm{C}[27$, 28]. The OB-fold structures of Sac7d and Sso7d are found in diverse binding molecules that recognize oligonucleotides, oligosaccharides, proteins, and metal ions [29-31]. Sso7d and related proteins have been employed in yeast, ribosome and phage display formats to select molecules that bind to diverse targets [23, 32-34].

Specific Mtb OTC binding variants with low nanomolar affinity were selected from an Sso7d library. The selected Sso7d variants were shown to bind to both folded and denatured recombinant Mtb OTC. Phage particles displaying the Sso7d variants were utilized to build a sandwich ELISA assay for Mtb OTC. The sandwich ELISA using intact phage particles paired with a purified monoclonal antibody has a limit of detection for recombinant Mtb OTC of 400 $\mathrm{pg} / \mathrm{mL}$, which is comparable to commercial antibody-based biosensors. The linear range of detection is $2 \mathrm{ng} / \mathrm{mL}$ to $125 \mathrm{ng} / \mathrm{mL}$. The phage-based sandwich ELISA maintains functionality at neutral and basic $\mathrm{pH}$ in the presence of urea and salt over the range of concentrations typically found in human urine. Selected binding variants maintained the high thermostability of the original Sso7d scaffold, with melting temperatures greater than $90{ }^{\circ} \mathrm{C}$.

\section{Materials and Methods}

All enzymes for cloning and mutagenesis were purchased from New England Biolabs (Ipswich, MA, USA). Unless otherwise noted, antibiotics were used at the following concentrations: ampicillin (amp) $100 \mu \mathrm{g} / \mathrm{mL}$; carbenicillin (carb) $50 \mu \mathrm{g} / \mathrm{mL}$, tetracycline (tet) 30 $\mu \mathrm{g} / \mathrm{mL}$, chloramphenicol (cam) $30 \mu \mathrm{g} / \mathrm{mL}$ and kanamycin (kan) $50 \mu \mathrm{g} / \mathrm{mL}$ (Sigma-Aldrich, St. Louis, MO, USA). All liquid cultures were shaken at $225 \mathrm{rpm}$ unless otherwise noted. All wash steps in ELISA and library panning are $300 \mu \mathrm{L}$, and 96-well plates were tapped on clean paper towels between each step to remove any remaining liquid in the wells. In this manuscript, all solutions containing phosphate-buffered saline (PBS) are made as $137 \mathrm{mM} \mathrm{NaCl}, 2.7 \mathrm{mM} \mathrm{KCl}$, $12 \mathrm{M} \mathrm{Na}_{2} \mathrm{HPO}_{4}$ and $1.2 \mathrm{mM} \mathrm{KH}_{2} \mathrm{PO}_{4}$ at $\mathrm{pH}$ 7.4. All oligonucleotides were purchased from IDT 
(Coralville, IA, USA). All constructs were verified by sequencing (Proteomics and Metabolomics Facility, Colorado State University).

\section{Purification of Recombinant Mtb Ornithine Transcarbamylase (Mtb OTC)}

The gene for Mtb OTC (Rv1656) was amplified from the H37Rv genome (BEI resources) by PCR with Oligos 1 and 2 (Table A.1). The PCR product was purified and extracted from an agarose gel $(0.8 \%$ in TAE) using a Qiagen gel extraction kit. The purified PCR product was cut using EcoRI and SapI and ligated into pTYB21 (New England Biolabs). The recombinant plasmid pNZB2 was verified by sequencing. Oligos 3 and 4 (Table A.1) were used to amplify the $M t b$ OTC gene from $\mathrm{pNZB} 2$. The PCR product was cut using NdeI and HindIII and ligated into pET23b (Novagen/EMD Millipore). The resulting vector pET23b-Rv1656 was verified by sequencing.

The $M t b$ OTC protein was expressed from pET23b-Rv1656 in E. coli BL21(DE3) pLysS (F, omp T, $h s d \mathrm{~S}_{\mathrm{B}}\left(\mathrm{r}_{\mathrm{B}}-, \mathrm{m}_{\mathrm{B}}-\right)$, dcm, gal, $\lambda(\mathrm{DE} 3), \mathrm{pLysS}, \mathrm{Cm}^{\mathrm{r}}$, Promega, Madison, WI, USA). A single colony was grown overnight in $30 \mathrm{~mL}$ of LB cam/amp at $37{ }^{\circ} \mathrm{C}, 125 \mathrm{rpm}$. The cells were harvested by centrifugation at $5000 \mathrm{x} g$ at room temperature. Cell pellets from the overnight culture were resuspended in $4 \mathrm{~L} \mathrm{LB}$ cam/amp and grown at $37{ }^{\circ} \mathrm{C}, 125 \mathrm{rpm}$. IPTG at $0.2 \mathrm{mM}$ was added to induce protein expression when the cell culture reached an $\mathrm{OD}_{600}$ of 0.5 . Protein expression proceeded at $25{ }^{\circ} \mathrm{C}$ and $125 \mathrm{rpm}$ overnight. Cells were harvested by centrifugation at $10000 \mathrm{xg}$ for $20 \mathrm{~min}$ at room temperature and resuspended in $20 \mathrm{~mL}$ of binding buffer $(20 \mathrm{mM}$ Tris- $\mathrm{HCl}, 500 \mathrm{mM} \mathrm{NaCl}, 5 \mathrm{mM}$ imidazole, $\mathrm{pH}$ 7.9). One-half cOmplete mini EDTA free protease inhibitor cocktail tablet (Roche), deoxyribonuclease I from bovine pancreas, type IV (Sigma-Aldrich) at a final concentration of $1.5 \mu \mathrm{g} / \mathrm{mL}$, and lysozyme (Sigma) at a final concentration of $200 \mu \mathrm{g} / \mathrm{mL}$ were added to the resuspended cell pellet. The cells were vortexed at room temperature for $15 \mathrm{~min}$ followed by sonication for 10 cycles of $60 \mathrm{sec}$ on and $90 \mathrm{sec}$ off (Qsonica). Insoluble debris was separated by centrifugation at $16000 \mathrm{xg}$ for $90 \mathrm{~min}$ at $4{ }^{\circ} \mathrm{C}$ resulting in $20 \mathrm{~mL}$ of clarified lysate. Samples from the clarified lysate and debris pellet were analyzed on an SDS-PAGE gel.

$M t b$ OTC was purified from the clarified lysate by FPLC using Ni-NTA slurry (Bio-Rad). After loading the clarified lysate, the column was washed with 10 column volumes (CV) of binding buffer $(20 \mathrm{mM}$ Tris- $\mathrm{HCl}, 500 \mathrm{mM} \mathrm{NaCl}, 5 \mathrm{mM}$ imidazole, $\mathrm{pH}$ 7.9). The column was rinsed with $6 \mathrm{CV}$ of wash buffer $(20 \mathrm{mM}$ Tris- $\mathrm{HCl}, 500 \mathrm{mM} \mathrm{NaCl}, 60 \mathrm{mM}$ imidazole, $\mathrm{pH}$ 7.9), followed by $10 \mathrm{CV}$ of $10 \mathrm{mM}$ Tris- $\mathrm{HCl}$, and $10 \mathrm{CV}$ of $0.5 \%$ ASB-14 (Calbiochem), $10 \mathrm{mM}$ Tris$\mathrm{HCl}$ to remove endotoxin. ASB-14 was removed from the column using $10 \mathrm{CV}$ of $10 \mathrm{mM}$ Tris$\mathrm{HCl}$. Mtb OTC was eluted using two CV of elution buffer $(10 \mathrm{mM}$ Tris-HCl, $1 \mathrm{M}$ imidazole, $\mathrm{pH}$ 8.0 prepared with endotoxin free water). The $M t b$ OTC elution was dialyzed against $4 \mathrm{~L}$ of 10 $\mathrm{mM}$ of ammonium bicarbonate buffer at $4{ }^{\circ} \mathrm{C}$ for 32 hours using a $3500 \mathrm{MW}$ dialysis membrane. The ammonium bicarbonate buffer was changed every 8 hours. Mtb OTC was quantified by Micro BCA protein assay (Thermo Scientific/Pierce). $32 \mathrm{mg}$ of purified $M t b$ OTC was obtained.

\section{Silver stain}

Recombinant $M t b$ OTC was visualized on a $10 \%$ polyacrylamide gel by silver stain. After electrophoresis, the gel was prepared by sequential agitation in the following: a solution of $40 \%$ 
aqueous methanol with $10 \%$ glacial acetic acid with for $45 \mathrm{~min}$, a solution of $5 \%$ aqueous methanol with $7 \%$ glacial acetic acid for $5 \mathrm{~min}$, and a solution of $2 \%$ aqueous gluteraldehyde for $5 \mathrm{~min}$. The gel was agitated 3 times for 10 minutes each in ultrapure water. The polyacrylamide gel was treated with a solution of $25 \mu \mathrm{g} / \mathrm{mL}$ dithiothreitol (Mallinckrodt) for $5 \mathrm{~min}$ and stained with a $0.1 \% \mathrm{AgNO}_{3}$ solution for $5 \mathrm{~min}$. Prior to visualization, the gel was rinsed twice with ultrapure water. Proteins were visualized using $60 \mathrm{~mL}$ developing solution $\left(6 \mathrm{~g} \mathrm{Na}_{2} \mathrm{CO}_{3}\right.$ and 3 drops of formaldehyde in $200 \mathrm{~mL}$ of ultrapure water). As soon as color began to appear on the gel, the developing solution was decanted. Fresh developing solution was added to the gel, and color was allowed to develop further. Color development was quenched by adding $20 \mathrm{~mL}$ of $50 \%$ aqueous citric acid. After a $30 \mathrm{~min}$ incubation, the gel was washed in ultrapure water for several hours.

\section{Preparation of Monoclonal Hybridoma Cells against Mtb OTC}

Two female BALB/c mice (National Cancer Institute) were immunized with four successive injections of purified recombinant $M t b$ OTC. For each of the first three injections, $50 \mu \mathrm{g}$ recombinant $M t b$ OTC were emulsified in $200 \mu \mathrm{L}$ of Freund's incomplete adjuvant (Sigma). For the fourth injection, $100 \mu \mathrm{g}$ recombinant $\mathrm{Mtb}$ OTC was dissolved in $0.9 \% \mathrm{NaCl}$. Sera of the immunized mice were evaluated for an immune response to Mtb OTC by ELISA and Western blot after the second, third, and fourth injections. The spleen of the mouse with the greater immune response was harvested on the fourth day after the fourth Mtb OTC injection. The harvested spleen was fused with SP2/0 myeloma cells in polyethylene glycol. The fused cells were transferred to 10 sterile 96-well tissue culture plates [35]. The antibodies secreted in each well were screened by ELISA against recombinant $M t b$ OTC. Culture supernatants from positive clones were evaluated by Western blot. Clones identified as binding to Mtb OTC by both ELISA and Western blot were subcloned in SP2/0 myeloma cells by limiting dilution. The subcloned cells were incubated for a week prior to evaluation by ELISA for binding to Mtb OTC and further verification by Western blot. This set of monoclonal hybridoma cell lines was frozen at $70{ }^{\circ} \mathrm{C}$.

\section{Purification of Mtb OTC Monoclonal Antibody}

Hybridoma cells were grown serially to a maximum volume of 2 L in RPMI medium until the $\mathrm{pH}$ dropped below 7.0. Culture supernatants were prepared by centrifugation at $2400 \mathrm{xg}$ for 20 minutes at $4{ }^{\circ} \mathrm{C}$. Purification of the $M t b$ OTC monoclonal antibody was carried out at $4{ }^{\circ} \mathrm{C}$ using a column loaded with 0.5 to $0.75 \mathrm{~mL}$ of Protein-G Sepharose (Sigma). The resin was washed with $20 \mathrm{~mL}$ of PBS. The culture supernatant was adjusted to $\mathrm{pH} 7.4$ with $10 \mathrm{M} \mathrm{NaOH}$ and loaded to the column at a rate of $1-2 \mathrm{~mL} / \mathrm{min}$. The flow-through was collected and reloaded to the column. The collecting and reloading process was repeated twice. The Protein-G Sepharose resin was washed with $50 \mathrm{~mL}$ PBS to remove all non-bound protein. $1 \mathrm{~mL}$ of $10 \mathrm{mM}$ ammonium bicarbonate was loaded to the column to prepare the column for elution. The bound $\mathrm{Mtb}$ OTC monoclonal antibody was eluted with $0.2 \mathrm{M}$ glycine- $\mathrm{HCl}, \mathrm{pH} 3.0$. All elution fractions were collected and neutralized with $10 \mathrm{mM}$ ammonium bicarbonate. BCA protein assay was used to identify elution fractions that contained antibody. Fractions containing antibody were combined. The concentration of the purified antibody was determined by BCA protein assay, and the purity was confirmed by polyacrylamide gel. The purified antibody was dialyzed against $2 \mathrm{~L}$ of $10 \mathrm{mM}$ ammonium bicarbonate in a $3500 \mathrm{MW}$ dialysis membrane at $4{ }^{\circ} \mathrm{C}$. The buffer was 
changed every 8 hours for 24 hours. Buffer exchanged Mtb OTC monoclonal antibody was lyophilized for long-term storage. Typical yields of purified antibody were $5-7.5 \mathrm{mg} / \mathrm{L}$ of culture supernatant.

\section{Sso7d Phage Displayed Library Construction via Kunkel Mutagenesis}

A phage displayed library of Sso7d variants was constructed by Kunkel mutagenesis as described previously [23]. Briefly, CJ236 E. coli $\left(\mathrm{F} \Delta\left(\right.\right.$ HindIII):: cat $\left(\mathrm{Tra}^{+} \mathrm{Pil}^{+} \mathrm{Cam}^{\mathrm{R}}\right) /$ ung-1 relA1 dut-1 thi-1 spoT1 mcrA) harboring the Sec-Sso7d-rArg template were infected with M13KO7 and grown overnight at $30{ }^{\circ} \mathrm{C}$ in the presence of excess uridine. Phage supernatants were prepared by centrifugation at $17,000 \mathrm{x} g$ to pellet the cells. Phage were precipitated by decanting the supernatant from the pelleted cells into $1 / 5^{\text {th }}$ volume polyethylene glycol (PEG) $8000 / 2.5 \mathrm{M} \mathrm{NaCl}$ in water [36]. The phage pellet was resuspended in $1 / 100^{\text {th }}$ of the original culture volume PBS. Single-stranded uridine-enriched (ss dU) DNA was isolated using the QIAprep Spin M13 kit (Qiagen). The inclusion of rare Arg codons in the library template is expected to reduce the translation of the starting template and place a metabolic burden on cells, leading to lower background in the library [37].

The library was generated using $100 \mu \mathrm{g}$ Sec-Sso7d-rArg ss dU DNA and a 10-fold molar excess of 5' phosphorylated Oligo 5 (Table A.1). The library was constructed using the degenerate codon NNC which encodes 15 of 20 amino acids (excluding Met, Lys, Glu, Gln, and Trp) and does not introduce any stop codons. The theoretical size of the library is $16^{9}=6.9 \mathrm{x}$ $10^{10}$ DNA sequences encoding $15^{9}=3.9 \times 10^{10}$ protein sequences. Following PCR spin kit cleanup and elution in ultrapure water, the library DNA was electroporated into SS320 cells ((MC1061 F', Lucigen) [F'proAB+lacIqlacZAM15 Tn10 (tetr)] hsdR mcrB araD139 $\triangle$ (araABCleu)7679 lacX74 galUgalK rpsL thi), resulting in $1.3 \times 10^{11}$ unique transformants. After transformation, cells were infected with $\mathrm{M} 13 \mathrm{KO} 7$ and transferred to $1 \mathrm{~L}$ of 2YT media with 30 $\mu \mathrm{g} / \mathrm{mL}$ tet, $60 \mu \mathrm{g} / \mathrm{mL}$ carb, and $10 \mu \mathrm{g} / \mathrm{mL}$ kan and grown overnight at $30{ }^{\circ} \mathrm{C}$. Phage particles were isolated by PEG precipitation. Aliquots of the library in PBS/14\% glycerol were stored at $80{ }^{\circ} \mathrm{C}$.

\section{Selection of binding variants}

Sso7d variants that bind Mtb OTC were selected by panning the phage library against purified protein. $1 \mu \mathrm{g}$ Mtb OTC was coated to MaxiSorp ${ }^{\circledR} 96$ well ELISA plates (Nunc Inc.) in $100 \mu \mathrm{L}$ coating buffer $\left(0.1 \mathrm{M} \mathrm{Na}_{2} \mathrm{CO}_{3}, 0.1 \mathrm{M} \mathrm{NaHCO}_{3}, \mathrm{pH} 9.6\right)$ at $4{ }^{\circ} \mathrm{C}$ overnight. Five target coated wells were used in the first round of selection; 4 wells were used in rounds 2-5. Wells were washed 3 times with PBS, pH 7.4. Wells were blocked using $300 \mu \mathrm{L}$ of blocking buffer (PBS with 4\% milk powder) for 1 hour at room temperature. Blocked wells were washed 6 times with $0.05 \%(\mathrm{v} / \mathrm{v})$ Tween 20 in PBS (PBST). In all rounds of panning, approximately $10^{13}$ library phages $(50 \mu \mathrm{L}$ of concentrated starting library phage or those amplified and concentrated from the previous round plus $50 \mu \mathrm{L}$ blocking buffer) were added to each well. After incubation for 2 hours at room temperature, unbound phage particles were discarded. Wells were washed to remove unbound phage particles; stringencies increased in each round of selection. After the first round of selection, wells were washed 3 times with PBS. Washes with PBST were added for subsequent rounds of panning. The second round of selection included 3 PBST washes followed by 2 PBS washes. The third round of panning included 6 PBST washes followed by 2 PBS 
washes. The fourth round of selection included 10 PBST washes followed by 2 PBS washes. The fifth round of selection included 20 PBST washes and the last two PBST washes were incubated for $15 \mathrm{~min}$, then followed by 2 PBS washes. Bound phages were eluted with a 25 minute incubation of $100 \mu \mathrm{L}$ of $100 \mathrm{mM}$ glycine $\mathrm{HCl}, \mathrm{pH} 2.2$ at room temperature. Eluents from multiple wells were combined and neutralized using 1.5 M Tris, $\mathrm{pH}$ 8.8.10 $\mu \mathrm{L}$ of the pooled neutralized phage eluent were titered (see below) to determine the output of each round of selection. The remainder of the pooled phages were propagated in $5 \mathrm{~mL}$ of NEB 5-alpha F' $E$.

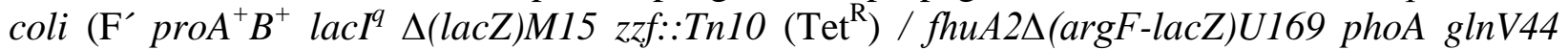
$\Phi 804$ (lacZ)M15 gyrA96 recAl relA1 endA1 thi-1 hsdR17). The infection was allowed to procced for 30 minutes without shaking followed by 30 minutes of incubation with shaking. Then the entire culture was added to $15 \mathrm{~mL} 2 \mathrm{YT}$ tet/carb with $3 \%$ glucose. M13KO7 (MOI of 50) was added when the culture reached an $\mathrm{OD}_{600} 0.5$. After two 30 minute incubations, first without shaking and then with shaking, cells were spun and resuspended in $100 \mathrm{~mL}$ of $2 \mathrm{YT}$ tet/carb/kan media without glucose, followed by overnight incubation at $30{ }^{\circ} \mathrm{C}$. Phages isolated by PEG precipitation and concentrated 100-fold were used for the following round of selection. Amplified phages were exposed to a blocked well during rounds 3 through 5 of selection to evaluate non-target protein specific phage retention.

\section{Phage Titration}

Phage output from each round of panning was determined using a 10-fold serial dilution series of the eluted phages and NEB 5-alpha F' cells grown to an $\mathrm{OD}_{600} 0.5$ at $37{ }^{\circ} \mathrm{C}$. After a 30 min incubation at $37^{\circ} \mathrm{C}$ for infection, at least $310 \mu \mathrm{L}$ spots for each phage dilution were plated on LB agar plates and incubated at $37{ }^{\circ} \mathrm{C}$ overnight. Phage titers were calculated from the dilution with the highest number of countable colonies.

\section{Enzyme-Linked Immunosorbent Assay (ELISA)}

For rounds of panning, $10 \mu \mathrm{g} M t b$ OTC diluted in coating buffer $(0.1 \mathrm{M}$ sodium carbonate, $0.1 \mathrm{M}$ sodium bicarbonate, $\mathrm{pH}$ 9.6) was coated to an ELISA plate at $4{ }^{\circ} \mathrm{C}$ overnight. The following day, wells were washed 3 times with PBS and blocked with blocking buffer at room temperature for 1 hour. Blocking buffer was removed, and wells were washed 6 times with PBST. Monoclonal antibody mAb-MtbOTC-1B8 or phage supernatants were diluted 1:1 in blocking buffer, added to the wells, and incubated at room temperature for 2 hours. Wells were washed 6 times with PBST. $100 \mu \mathrm{L}$ of anti-M13 antibody conjugated with HRP (Thermo Scientific/Pierce, 1:5000 dilution in blocking buffer) or anti-mouse antibody conjugated with HRP (Thermo Scientific/Pierce, 1:5000 dilution in blocking buffer) were added to each well and incubated at room temperature for 1 hour. Wells were washed 6 times with PBST and 2 times with PBS. Colorimetric assays were performed using 1-Step Ultra TMB ELISA Substrate (Thermo Scientific/Pierce). The reaction was quenched by addition of $2 \mathrm{M}$ sulfuric acid after a 30 minute incubation at room temperature. Absorbance at $450 \mathrm{~nm}$ was determined using a Biotek Synergy H1 microplate reader.

\section{Binding Analysis of Individual Clones}

Clones were evaluated by picking individual colonies into $150 \mu \mathrm{L}$ of $2 \mathrm{YT}$ tet/carb with $3 \%$ glucose in a 96 well plate incubated at $37{ }^{\circ} \mathrm{C}$ overnight. The following day, $5 \mu \mathrm{L}$ of each 
overnight culture was transferred to $150 \mu \mathrm{L}$ of $2 \mathrm{YT}$ tet/carb with $3 \%$ glucose in a fresh 96 well plate. Cultures were allowed to regrow to $\mathrm{OD}_{600} 0.5$ at $37{ }^{\circ} \mathrm{C}$ (generally about 2.5 hours). M13KO7 was added to each well at a MOI of 20. After incubation at $37{ }^{\circ} \mathrm{C}$ for $30 \mathrm{~min}$, the cells were pelleted by centrifugation at $5000 \mathrm{xg}$ at room temperature and resuspended in $2 \mathrm{YT}$ tet/carb/kan without glucose. The 96-well plate was incubated with shaking at $30{ }^{\circ} \mathrm{C}$ overnight. Cells were pelleted at 17,000 $\mathrm{xg}$, and the phage supernatants were used in an ELISA assay as described above to characterize the binding affinity of each phage displayed Sso7d variant. Clones whose phage supernatants displayed ELISA signals in wells coated with $500 \mathrm{ng} M t b$ OTC at least 3-fold greater than that in blocked wells were considered hits. In actuality, each of the positive ELISA signals were at least 43 -fold greater than background.

\section{Western Blot}

After separation on a polyacrylamide gel by electrophoresis, purified recombinant $M t b$ OTC was transferred to a $0.2 \mu \mathrm{m}$ nitrocellulose membrane (Bio-Rad) by electrophoresis at $50 \mathrm{~V}$ for 1 hour using transfer buffer (25 mM Tris base, $190 \mathrm{nM}$ glycine and $20 \%$ aqueous ethanol). The nitrocellulose membrane was blocked in blocking buffer for 1 hour and rinsed three times with PBST. The membrane was cut into strips, and strips were incubated with Mtb OTC antibody mAb-MtbOTC-1B8 or phage particles displaying Sso7d-MtbOTC binding variants in blocking buffer overnight at room temperature with agitation. The next morning, the membrane strips were subjected to 6 consecutive 15 minute PBST washes. Secondary antibody, anti-M13 antibody conjugated with HRP (1:5000 dilution in PBST) or anti-mouse antibody conjugated with HRP (1:5000 dilution in PBST), was added to each membrane strip and allowed to incubate for 1.5 hours. The membrane strips were subjected to 6 consecutive 15 minute PBST washes, followed by two washes with PBS to remove the Tween 20. The CN/DAB substrate kit (Thermo Scientific/Pierce) was used to visualize bound protein.

\section{Competitive ELISA to Determine Binding Affinity}

ELISA signals at different concentrations of both immobilized target protein and purified phage were used to determine a set of conditions which led to $50 \%$ saturation of the ELISA signal. Wells for competitive ELISA were prepared as described above for the phage supernatant ELISA using $62.5 \mathrm{ng} /$ well of immobilized Mtb OTC. $6.8 \times 10^{10} \mathrm{Sso} 7 \mathrm{~d}-\mathrm{MtbOTC}-2 \mathrm{H} 8$ phage particles were pre-equilibrated with concentrations free $M t b$ OTC across four orders of magnitude $\left(10^{-10}-10^{-6} \mathrm{M}\right)$ in blocking buffer for 2 hours at room temperature. A sample of Sso7d-MtbOTC-2H8 phage that was not pre-equilibrated with Mtb OTC served as a reference for the $100 \%$ phage-bound absorbance. Phage solutions were added to each prepared well and allowed to bind for 15 minutes. ELISA was performed and visualized as described above. Absorbances at $450 \mathrm{~nm}$ for each concentration of competitor were normalized to the absorbance for the phage sample with no competitor. Data were plotted as relative absorbance at $450 \mathrm{~nm}$ versus concentration of competitor. Dissociation constants were determined by fitting the data using a 4-parameter logistic equation (Equation A.1). Measurements were performed in triplicate for each experiment; the $K_{D}$ reported represents the average of three independent experiments.

\section{Sandwich ELISA}


Purified Sso7d-MtbOTC-2H8 protein, Sso7d-MtbOTC-2H8 phage particles, and mAbMtbOTC-1B8 antibody were coated to each well at $4{ }^{\circ} \mathrm{C}$ overnight. For purified Sso7dMtbOTC-2H8 protein and mAb-MtbOTC-1B8 antibody, the coating buffer was composed of 0.1 $\mathrm{M}$ sodium carbonate and $0.1 \mathrm{M}$ sodium bicarbonate at $\mathrm{pH}$ 9.6. For Sso7d-MtbOTC binding variant phage solutions, the coating buffer was PBS. The coated wells were washed 3 times with PBST and blocked with blocking buffer at room temperature for 1 hour. After blocking, wells were washed 5 times with PBST. Mtb OTC in blocking buffer was added to each well and allowed to bind for 2 hours. Unbound $M t b$ OTC was removed by washing wells 4 times with PBST. mAb-MtbOTC-1B8 antibody or Sso7d-MtbOTC binding variant phage particles diluted in blocking buffer were added to each well and incubated for 2 hours. Wells were washed 4 times with PBST prior to addition of anti-M13 antibody conjugated with HRP (1:3000 dilution in blocking buffer) or anti-mouse antibody conjugated with HRP (1:3000 dilution in blocking buffer). After 1 hour of incubation at room temperature, the unbound secondary antibody was removed by washing wells 5 times with PBST and 2 times with PBS. Visualization proceeded as described above.

\section{Heat Tolerance of Sso7d Variants on Phage}

The heat tolerance of phages displaying Sso7d variants that bind Mtb OTC was evaluated by ELISA. Phage stock solutions were incubated at temperatures between 50 and $90{ }^{\circ} \mathrm{C}$ for $30 \mathrm{~min}$. Control samples were incubated on ice for the duration of each experiment. After exposure to elevated temperatures, all phages were kept at room temperature for 1 hour. Phage solutions were diluted 300-fold in blocking buffer prior to performing ELISA as described above in wells coated with $1 \mu \mathrm{g}$ of $M t b$ OTC.

\section{Preparation and Purification of Sso7d Variants Off Phage}

Oligos 6 and 7 (Table A.1) were used to amplify Sso7d variants for cloning into a pET23b protein expression vector using NdeI and HindIII restriction sites. pET23b-Sso7d variants were verified by sequencing. Vectors were transformed into BL21(DE3) pLysS cells by electroporation. 1.2 L of LB cam/carb were inoculated from an overnight culture and incubated at $37^{\circ} \mathrm{C}$. Protein expression was induced by addition of IPTG to a final concentration of $0.5 \mathrm{mM}$ when the large culture reached an $\mathrm{OD}_{600}$ of 0.5 . Cells were grown overnight at $30{ }^{\circ} \mathrm{C}$. Cells were harvested by centrifugation and lysed by sonication. Sso7d variants were purified from the clarified lysate by Ni-NTA affinity chromatography on a BioLogic LP FPLC (BioRad, Hercules, CA, USA). Eluted proteins were dialyzed against PBS and quantified by Micro BCA protein assay. Yields of each Sso7d variant were typically between 30 and $40 \mathrm{mg} / \mathrm{L}$.

\section{Differential Scanning Calorimetry (DSC)}

DSC experiments were performed on a Nano DSC model 6300 (TA Instruments). Sso7d variants were buffer exchanged into PBS and diluted to a final concentration of approximately $0.5 \mathrm{mg} / \mathrm{mL}$. All samples were degassed under vacuum. Both the reference cell and the sample cell were rinsed with degassed PBS 3 times. Degassed PBS was used for background buffer scans. Two scan cycles, each consisting of one heating and one cooling cycle from $5{ }^{\circ} \mathrm{C}$ to 110 ${ }^{\circ} \mathrm{C}\left(1{ }^{\circ} \mathrm{C} / \mathrm{min}\right)$ were performed for each Sso7d variant. Data were recorded in DSC Run software 
(TA Instruments) and analyzed using the two state scale model in NanoAnalyze (TA Instruments). Each Sso7d variant was scanned twice.

\section{Results and Discussion}

\section{Purification of Recombinant Mtb OTC}

The $M t b$ gene Rv1656 coding for ornithine transcarbamylase (Mtb OTC) was amplified from strain H37Rv genomic DNA and ligated into vector pET23b, appending a hexahistidine tag to the C-terminus. The protein was expressed in BL21(DE3)pLysS cells and purified by Ni-NTA column. The protein purity and yield were characterized by SDS-PAGE and BCA protein assay. The silver stained gel showed a single sharp band at a molecular weight of $37 \mathrm{kDa}$, the theoretical molecular weight of the purified recombinant Mtb OTC protein. Loading $2 \mu \mathrm{g}$ of purified $M t b$ OTC showed no other protein bands, suggesting high purity (as the detection level is approximately $1 \mathrm{ng}$ for silver staining, Figure 1a). The yield of purified $M t b$ OTC was $8 \mathrm{mg} / \mathrm{L}$ of culture.

\section{Library Selection against Purified Recombinant Mtb OTC}

Variants of the Sso7d scaffold protein that bind purified recombinant Mtb OTC were selected from a previously-described Sso7d phage display library through 5 rounds of panning [23]. The library includes extensive amino acid side chain diversity at 9 positions within the 3 antiparallel beta sheets that comprise the DNA-binding interface of Sso7d (Figure 2). The library was constructed using the degenerate codon NNC which encodes 15 of 20 amino acids (excluding Met, Lys, Glu, Gln, and Trp) and does not introduce any stop codons. The theoretical size of the library is $16^{9}=6.9 \times 10^{10}$ DNA sequences encoding $15^{9}=3.9 \times 10^{10}$ protein sequences. The mutation efficiency was evaluated by analyzing XbaI digests of PCR products from 95 isolated colonies. The amplified fragment from a library member is not cut by XbaI, while the unmodified rare Arg template is digested. 12 of 95 colonies were identified as library members. DNA sequencing confirmed that each of those variants were unique library members. Considering mutagenic efficiency, the library contained $1.6 \times 10^{10}$ individual variants.

An increase in the titer of output phage is typically observed during panning experiments as an indication of the enrichment of binding variants. When the phage displayed Sso7d variant library was panned against several other protein targets, four of which are previously reported, output titers increased with each round of selection, as did the ratio of output titer between target-coated and blocked wells [23]. Although output titers did not increase when panning against $M t b$ OTC in this study, the ratio of output titer between target-coated and blocked wells did increase. ELISA evaluation of 71 clones after the fourth round of panning indicated that binding clones were selected (Table 1). Seven of these colonies showed selective binding, with ELISA signals 60-fold higher than background.

A fifth round of panning was performed; interestingly none of the 35 colonies analyzed showed ELISA signals greater than three-fold over background. Close inspection of the plated cells from the third through fifth rounds of panning revealed that two colony sizes were present. All of the colonies from the output phage titer plates from the fifth round were of similar size and colony morphology. However, two different sizes and transparencies of colonies were observed 
on plates from the third and fourth rounds of panning: smaller, transparent colonies and larger opaque colonies resembling those observed in the fifth round. ELISA signals from 18 of 24 phage supernatants produced from specifically-chosen small colonies off of the third round phage titer plate ranged from 47 -fold to 83 -fold over background.

The observed morphology difference suggests that the expression of functional phage fusion proteins inhibits cell growth. Similar growth effects were not observed when the same Sso7d library was panned against multiple other targets, suggesting that the growth effects on cells may be a particular feature of the variants that bind Mtb OTC [23]. Phage display selections typically balance the effects of enrichment through selective binding and enrichment through selective growth effects during the amplification of the library pool between panning steps. Generally, the selective binding enrichment offsets any growth effect difference in the library. Growth effects have been found to be a major factor in the selection of "commonly observed sequences" in phage display using commercially-available small peptide libraries [38, 39]. Our library was constructed employing a starting template containing multiple rare arginine codons rather than the more commonly employed stop codons. The purpose of the rare arginine variant was to increase the burden on cells containing the unmodified template. Positive growth effects as a result of not translating a fusion protein have been anecdotally implicated in failed phage display experiments.

\section{Sequence Analysis of the Sso7d Mtb OTC Binding Variants}

All of the ELISA-positive clones showed strong signals in wells containing target and signals identical to background against blocked wells, suggesting specific binding to Mtb OTC. The 12 clones with the highest ELISA signals were sequenced, yielding eight unique Sso7d variants (Table 2). Two sequences representing four of twelve sequenced clones were very similar. Clones Sso7d-MtbOTC-5B5 and Sso7d-MtbOTC-5A6 were identical at four of nine varied positions and contained similar amino acids at three additional positions (two $\mathrm{F}$ vs $\mathrm{Y}$ and one $\mathrm{T}$ vs V). Both sequences also contained appropriately positioned cysteine residues, suggesting an identical disulfide bond. A positive residue at closely spaced positions, either the end or beginning of adjacent antiparallel beta strands (R32 vs R40), was identified in both sequences. Sequences Sso7d-MtbOTC-4A8 and Sso7d-MtbOTC-5A1 also showed a high degree of similarity. Sso7d-MtbOTC-4A8 and Sso7d-MtbOTC-5A1 were identical at three of nine positions and had similar amino acids at four of nine positions (two F vs. Y, one F vs L, and one $\mathrm{Y}$ vs L). The remaining four sequences did not display strong similarity either to each other or to the Sso7d-MtbOTC-5B5/Sso7d-MtbOTC-5A6 or Sso7d-MtbOTC-4A8/Sso7d-MtbOTC-5A1 pairs.

\section{Analysis of Binding Strength and Mode of Sso7d-MtbOTC Binding Variants}

A two-fold dilution series of purified phage particles representing each Sso7d-MtbOTC binding variant sequence was used in a series of phage ELISAs in order to quickly screen their binding performance (Figure 3). ELISA wells were coated with the same concentration of Mtb OTC, and the concentration of phage particle added to each well was varied. The binding strengths for each Sso7d-MtbOTC binding variant appear to be similar; only about 10-fold more Sso7d-MtbOTC-2H12, the apparent weakest binder, was required to achieve an ELISA signal 
similar to that of Sso7d-MtbOTC-2H8, the apparent tightest binder. Repeat analysis of phage dilutions showed similar results.

Binding performance is not the sole requirement of a molecule in a biosensor. An important consideration is whether the binding epitope is contiguous in sequence or requires the folded form of the target protein. Generally, binding molecules that recognize epitopes that do not depend on the secondary or tertiary structure of the target protein have greatest potential as diagnostic molecules. The dependence of the presentation of binding epitopes on the folding state of $M t b$ OTC for the eight identified Sso7d-MtbOTC binding variants and a monoclonal antibody mAb-MtbOTC-1B8 were investigated by Western blot (Figure 1b). The monoclonal antibody was generated by immunization of mice with recombinant Mtb OTC. All eight Sso7d variants and $\mathrm{mAb}-\mathrm{MtbOTC}-1 \mathrm{~B} 8$ bind to the denatured form of recombinant Mtb OTC. The sharp bands at $40 \mathrm{kDa}$ in the Western blot indicate binding to intact Mtb OTC. The presence of bands at $25 \mathrm{kDa}$ and $35 \mathrm{kDa}$ are attributed to partially-degraded $M t b$ OTC because these bands became apparent after storage of the recombinant protein and were not detected via silver staining during the original isolation of $M t b$ OTC. The selected Sso7d variants and mAb-MtbOTC-1B8 show different levels of interaction with the intact and degraded protein bands. mAb-MtbOTC-1B8 only shows a band at the intact protein molecular weight, while Sso7d-MtbOTC-5B5/Sso7dMtbOTC-5A6 and to a lesser extent Sso7d-MtbOTC-5B4, Sso7d-MtbOTC-5B7 and Sso7dMtbOTC-4A8 show bands at $40 \mathrm{kDa}$ and $25 \mathrm{kDa}$. The interaction of some but not all of the Sso7d variants with the degraded protein fragments suggests that Sso7d variants recognizing different epitopes may have been selected.

\section{Analysis of Binding Affinity and Thermal Stability of Sso7d-MtbOTC-2H8}

The binding affinity of Sso7d-MtbOTC-2H8 phage, the apparent tightest binder, was evaluated by competitive ELISA. Conditions for the competitive ELISA were established by identifying the concentrations of both the coated Mtb OTC and Sso7d-MtbOTC-2H8 phage particle that led to $50 \%$ saturation of the ELISA signal. Nanomoloar to micromolar concentrations of soluble, competing $M t b$ OTC were equilibrated with the identified quantity of Sso7d-MtbOTC-2H8 displaying phage particles before addition to Mtb OTC coated ELISA wells. The $K_{D}$ was estimated to be $22.8 \pm 2.4 \mathrm{nM}$ based on the concentration of competitor that led to a $50 \%$ reduction in the ELISA signal (Figure 4).

The extreme thermal stability of Sso7d is one of its most appealing properties as a molecular scaffold and is potentially of great utility in designing point-of-care diagnostics. M13 phage particles themselves also have high thermal stability [23, 40]. The tolerance of the Sso7dMtbOTC-2H8 phage particles to elevated temperatures was evaluated by incubating the particles at temperatures between $50{ }^{\circ} \mathrm{C}$ to $90{ }^{\circ} \mathrm{C}$ for 30 minutes. After heating, the phages were cooled on ice, and the Mtb OTC binding ability was evaluated by ELISA. Samples of phage particles not exposed to elevated temperatures were evaluated as a control. The Sso7d-MtbOTC-2H8 phage retained binding to $M t b$ OTC after exposure to $70{ }^{\circ} \mathrm{C}$ for $30 \mathrm{~min}$, maintaining about $80 \%$ of the binding of unheated controls (Figure 5). After incubation at $80^{\circ} \mathrm{C}$, no binding was detected. The stability of the M13 phage particles was monitored by titer. Extended incubations up to $70{ }^{\circ} \mathrm{C}$ had no effect on the phage titer; phage titer decreased 10-fold after exposure to $80{ }^{\circ} \mathrm{C}$. Exposing phages to $90{ }^{\circ} \mathrm{C}$ decreased the titer to less than $10^{4} \mathrm{cfu} / \mathrm{mL}$, the detection limit of the assay. The Sso7d-MtbOTC-2H8 protein appears to be as stable as the highly stable phage particles. 
The melting temperature of Sso7d-MtbOTC-2H8 protein expressed and purified in the absence of the phage context was $91{ }^{\circ} \mathrm{C}$ as measured by DSC. Repeated temperature scanning suggested the folding of Sso7d-MtbOTC-2H8 was non-reversible in PBS at pH 7.4. The wildtype Sso7d protein is a model two-state folding protein, but several selected binding variants against different targets have shown irreversible denaturation.[23, 32]

\section{Assembly of a Sandwich ELISA for Mtb OTC Detection}

Sandwich ELISA is a well-established diagnostic method typically employing antibody reagents. In recent years, phage particles have been proposed as potential replacements for antibodies in diagnostics [41-43]. Diagnostic assays utilizing phage particles have significant potential advantages over antibody-based systems. Because multiple binding and signaling components of sensors may be synthesized and assembled as part of the phage life cycle process, phage-based assays have the potential to be inexpensive to manufacture. Moreover, because phage-based components are potentially highly multivalent, phage components have the potential to increase the sensitivity of interactions relative to antibodies. High sensitivity is expected based on the inherent binding strengths of selected interacting molecules extended by avidity effects of having multiple interacting sites connected to the same particle. Additionally, phage particles have been shown to increase the stability of proteins appended to them, and this effect may the extend shelf life of phage-based diagnostics [44].

Sso7d-MtbOTC-2H8 phage and the mAb-MtbOTC-1B8 antibody were used to construct an $M t b$ OTC ELISA asaay. In one scenario, the monoclonal antibody mAb-MtbOTC-1B8 was coated to the well, and Mtb OTC was detected using Sso7d-MtbOTC-2H8 phage particles (Figure 6a). In a second scenario, the phage and Mtb OTC monoclonal antibody were flipped; $M t b$ OTC was captured by immobilizing Sso7d-MtbOTC-2H8 phage in the ELISA well and detected using mAb-MtbOTC-1B8 (Figure 6b). The ELISA signal as a result of utilizing the mAb-MtbOTC-1B8 antibody to capture Mtb OTC was 4-fold higher than in the alternative ELISA design using the Sso7d-MtbOTC-2H8 phage as the capture reagent (Figure 6c). These results are most likely related to differences in the amount of binding molecules that can be immobilized in the ELISA wells in the different formats (e.g. on phage or as free antibodies) or a signal amplification that occurs when phage are employed for detection. Efficiencies of recognition of the target protein or differences in the properties of the secondary antibodies may also contribute to the response. The anti-M13 antibody binds to the major coat protein $\mathrm{p} 8$ of which there are 2700 copies per phage particle. Some signal amplification may result from the detection of Sso7d-MtbOTC-2H8 displaying phage, as opposed to employing the anti-mouse antibody which binds to the Fc region of the mAb-MtbOTC-1B8 antibody. Presumably only a single detection protein conjugate can bind to an immobilized antibody molecule.

The ELISA signal increased nearly 5-fold upon increasing the number of Sso7d-MtbOTC2H8 phage particles immobilized in the wells as the capture reagent (Figure 6c, blue bars). This observation suggests that at these concentrations, the ELISA wells may not be saturated with $M t b$ OTC binding moieties. The solubility and size of phage particles may limit the number of binding moieties that can be immobilized for antigen detection. In order to evaluate whether the amount of Sso7d-MtbOTC binding variant immobilized in the ELISA well as a result of being linked to a phage particle was contributing to the reduced signal, the capture abilities of the purified Sso7d-MtbOTC-2H8 protein and Sso7d-MtbOTC-2H8 phage were compared (Figure 7). 
Interestingly, the ELISA signal for wells in which phage particles were used to capture the $M t b$ OTC remained higher than that of the wells in which purified Sso7d-MtbOTC-2H8 protein was used as the capture reagent. Furthermore, the ELISA signal of the purified Sso7d-MtbOTC-2H8 did not increase when the amount of purified Sso7d-MtbOTC-2H8 applied to coat the ELISA well was increased from $1 \mu \mathrm{g}$ to $10 \mu \mathrm{g}$, suggesting that $1 \mu \mathrm{g}$ of purified Sso7d-MtbOTC-2H8 protein was enough to saturate the ELISA well surface. Together, these results suggest that phage particles might serve as a useful matrix to maintain the Sso7d-MtbOTC-2H8 binding structure and accessibility to the target protein.

Several factors may contribute to the observation that fewer Sso7d-MtbOTC-2H8 binding equivalents in the context of phage lead to a higher signal response than is observed for Sso7dMtbOTC-2H8 binding equivalents in the absence of the phage context (on the order of $1.1 \mathrm{pmol}$ on phage compared to 114 pmol off phage). The structure of purified Sso7d-MtbOTC-2H8 protein may have been altered as a result of the hydrophobic binding interactions required to immobilize the protein in the ELISA well. One of the attractive features of Sso7d is its small size, and that, coupled with a surface charge may have reduced the binding and accessibility of free Sso7d protein in the ELISA assay. Additionally, the blocking buffer used in each ELISA is composed of milk power, which contains approximately $80 \%$ casein, a protein that is much larger than Sso7d (molecular weight of $19 \mathrm{kDa}$ to $25 \mathrm{kDa}$ compared to $7 \mathrm{kDa}$ ). Alternatively, removal of the Sso7d-MtbOTC-2H8 protein from the phage context may reduce the binding affinity of the protein.

\section{Standard Curve for Recombinant Mtb OTC Detection}

Given the observation that the highest ELISA signals resulted from wells in which the mAbMtbOTC-1B8 antibody was used as the capture reagent and Sso7d-MtbOTC binding variant phage particles were utilized as the detection reagent, a standard curve for the assay was made to evaluate both the linearity of response and the limit of detection of Mtb OTC. ELISA wells were prepared by coating $11 \mu \mathrm{g}$ of mAb-MtbOTC-1B8 antibody. After washing and blocking the wells, 2-fold serial dilutions of Mtb OTC from $0 \mathrm{ng}$ to $200 \mathrm{ng}$ were added to each well. Mtb OTC was detected by adding $9 \times 10^{11}$ cfu of Sso7d-MtbOTC-2H8 phage to each well. A secondary antibody, anti-M13 ab/HRP and TMB were added to the wells to generate signal. The absorbance at $450 \mathrm{~nm}$ was measured and plotted as a function of $M t b$ OTC concentration (Figure 9). The signal was linear over recombinant $M t b$ OTC concentrations from $2 \mathrm{ng} / \mathrm{mL}$ to $125 \mathrm{ng} / \mathrm{mL}$ $\left(\mathrm{R}^{2}=0.9973\right)$. The limit of detection is estimated to be $400 \mathrm{pg} / \mathrm{mL}$. This Sso7d displaying phage particle sandwich ELISA is comparable with commercially-available antibody-based affinity assays, which typically have detection limits in the picomolar range [45, 46].

\section{Assay Tolerance to Urea, Ionic Stress and pH}

The linear range and limit of detection were measured in phosphate buffered saline. A useful point-of-care diagnostic for the detection of $M t b$ OTC must maintain function in a urine matrix. The extent to which the three major compositional variables of urine affect the assay detection sensitivity were evaluated. The composition of urine is widely variable and depends on diet, hydration, and kidney function [47]. In general, human urine contains $155 \mathrm{mM}-388 \mathrm{mM}$ urea, $69 \mathrm{mM}-258 \mathrm{mM}$ positively charged ions (mostly $\mathrm{Na}^{+}$and $\mathrm{K}^{+}$), $53 \mathrm{mM}-237 \mathrm{mM}$ negatively charged ions (mostly $\mathrm{Cl}^{-}$) and has a $\mathrm{pH}$ between 5.5 and 7.[47] A three factor, three level 
fractional factorial experimental design was utilized to investigate the tolerance of the Mtb OTC assay to the concentration of urea, ionic strength and $\mathrm{pH}$. For each factor, 3 compositions of synthetic buffer were selected to cover the range typically found in human urine (Table 3 ). Nine individual experiments were used to span the space of the 27 possible combinations of the three compositional variables [48]. The effect of each factor on assay performance was evaluated using a one-way analysis of variance (ANOVA) to quantify the significance, $p$ value $\leq 0.05$.

The ELISA signal for each of the 9 experiments was normalized to the assay performance in PBS buffer (Figure 10). For 6 of the 9 experiments, ELISA signals were essentially identical to those observed for operation in PBS. The 6 experiments for which assay performance was indistinguishable from the PBS control span the space of urea and salt concentrations in human urine. ELISA signals for the remaining three simulated urine scenarios were reduced to $23 \%$, $66 \%$, and $53 \%$ of the PBS reference. Each of those assays were performed at pH 5.0, which suggests that assay performance is adversely affected by acidic conditions. The tolerance to urea and salt in the range of urine is promising for creating an assay to diagnose active TB using unprocessed urine. Although at acidic $\mathrm{pH}$ the assay performance is reduced, sample $\mathrm{pH}$ may be easily adjusted by employing an assay framework in which the urine passes through a buffer impregnated matrix prior to evaluation (e.g. in a lateral flow set-up).

\section{Conclusions}

The challenge of frontline TB diagnosis is that high sensitivity and accuracy are required in a robust, field-stable format that is economically accessible in the most resource-limited areas of the world. TB detection using urine is actively being investigated, and Mtb OTC is a promising urine biomarker of active TB infection. We selected 8 different tight binding $M t b$ OTC proteins from a phage display library built upon the hyperthermostable Sso7d scaffold. The binding epitopes of the selected Sso7d variants do not appear to depend on the folded structure of the target protein. The apparent tightest binder, Sso7d-MtbOTC-2H8, has a $\mathrm{K}_{\mathrm{D}}$ of $22.8 \mathrm{nM}$ and was utilized to construct a sandwich ELISA assay in combination with a monoclonal antibody raised against Mtb OTC, mAb-MtbOTC-1B8. The sandwich ELISA assay shows a linear response between $2 \mathrm{ng} / \mathrm{mL}$ and $125 \mathrm{ng} / \mathrm{mL}$ recombinant $M t b$ OTC. The limit of detection is estimated to be $400 \mathrm{pg} / \mathrm{mL}$ recombinant $M t b$ OTC. Additionally, the sandwich ELISA assay is tolerant to urea and ionic stress in the concentration ranges typically seen in urine, suggesting that the assay is promising for the diagnosis of TB using unprocessed urine. Synthetic urine $\mathrm{pH}$ did significantly affect the sensitivity of the assay, but in a point-of-care environment $\mathrm{pH}$ may be easily adjusted by including a buffering agent as a component of the matrix supporting the assay. Replacement of antibodies by phage particles in sandwich ELISA assays has the potential to reduce cost without sacrificing detection sensitivity and specificity. With a sensitive assay in place, experiments to quantify the $M t b$ OTC in TB patients' urine and to validate $M t b$ OTC as a diagnostic of active tuberculosis infection are possible.

\section{Acknowledgements:}

We are extremely grateful to the laboratory of P. Shing Ho (Department of Biochemistry and Molecular Biology at Colorado State University) for assistance, expertise, and access to the DSC instrumentation. We thank Andrew Bradbury for a generous gift of plasmids. DNA sequencing was performed in the PMF at Colorado State University. This work was generously supported by 
the Bill and Melinda Gates Foundation, the Boettcher Foundation, and Colorado State University (N.Z., M.A.S, and J.D.F). 


\section{References}

1. WHO. (2015) Global tuberculosis report 2015, Geneva: World Health Organization, 2015.

2. WHO. (2014) Global tuberculosis report 2014, Geneva: World Health Organization, 2014.

3. Storla, D. G., Yimer, S., and Bjune, G. A. (2008) A systematic review of delay in the diagnosis and treatment of tuberculosis, BMC Public Health 8, 9.

4. Perkins, M. D., and Kritski, A. L. (2002) Diagnostic testing in the control of tuberculosis, Bull. World Health Organ. 80, 512-513.

5. $\quad$ Keeler, E., Perkins, M. D., Small, P., Hanson, C., Reed, S., Cunningham, J., Aledort, J. E., Hillborne, L., Rafael, M. E., Girosi, F., and Dye, C. (2006) Reducing the global burden of tuberculosis: The contribution of improved diagnostics, Nature suppl. 1, 4957.

6. McNerney, R., and Daley, P. (2011) Towards a point-of-care test for active tuberculosis: Obstacles and opportunities, Nat. Rev. Microbiol. 9, 204-213.

7. Cheon, S. A., Cho, H. H., Kim, J., Lee, J., Kim, H. J., and Park, T. J. (2016) Recent tuberculosis diagnosis toward the end TB strategy, J. Microbiol. Methods 123, 51-61.

8. Lawn, S. D. (2012) Point-of-care detection of lipoarabinomannan (LAM) in urine for diagnosis of HIV-associated tuberculosis: A state of the art review, BMC Infect. Dis. 12, 103.

9. Gaylord, H., Brennan, P. J., Young, D. B., and Buchanan, T. M. (1987) Most mycobacterium-leprae carbohydrate-reactive monoclonal-antibodies are directed to lipoarabinomannan, Infect. Immun. 55, 2860-2863.

10. Tucci, P., Gonzalez-Sapienza, G., and Marin, M. (2014) Pathogen-derived biomarkers for active tuberculosis diagnosis, Front. Microbiol. 5, 549.

11. Boehme, C., Molokova, E., Minja, F., Geis, S., Loscher, T., Maboko, L., Koulchin, V., and Hoelscher, M. (2005) Detection of mycobacterial lipoarabinomannan with an antigen-capture ELISA in unprocessed urine of Tanzanian patients with suspected tuberculosis, Trans. Roy. Soc. Trop. Med. Hyg. 99, 893-900.

12. Gounder, C. R., Kufa, T., Wada, N. I., Mngomezulu, V., Charalambous, S., Hanifa, Y., Fielding, K., Grant, A., Dorman, S., Chaisson, R. E., and Churchyard, G. J. (2011) Diagnostic accuracy of a urine lipoarabinomannan enzyme-linked immunosorbent assay for screening ambulatory HIV-infected persons for tuberculosis, J. Acquir. Immune Defic. Syndr. 58, 219-223.

13. Lawn, S. D., Edwards, D. J., Kranzer, K., Vogt, M., Bekker, L.-G., and Wood, R. (2009) Urine lipoarabinomannan assay for tuberculosis screening before antiretroviral therapy diagnostic yield and association with immune reconstitution disease, AIDS 23, 18751880.

14. Lawn, S. D., Kerkhoff, A. D., Vogt, M., and Wood, R. (2012) Diagnostic accuracy of a low-cost, urine antigen, point-of-care screening assay for HIV-associated pulmonary tuberculosis before antiretroviral therapy: A descriptive study, Lancet Infect. Dis. 12, 201-209.

15. Shah, M., Variava, E., Holmes, C. B., Coppin, A., Golub, J. E., McCallum, J., Wong, M., Luke, B., Martin, D. J., Chaisson, R. E., Dorman, S. E., and Martinson, N. A. (2009) Diagnostic accuracy of a urine lipoarabinomannan test for tuberculosis in hospitalized patients in a high HIV prevalence setting, J. Acquir. Immune Defic. Syndr. 52, 145-151. 
16. Dube, D. H., and Bertozzi, C. R. (2005) Glycans in cancer and inflammation. Potential for therapeutics and diagnostics, Nat. Rev. Drug Discov. 4, 477-488.

17. Jacobsen, M., Mattow, J., Repsilber, D., and Kaufmann, S. H. E. (2008) Novel strategies to identify biomarkers in tuberculosis, Biol. Chem. 389, 487-495.

18. Parida, S. K., and Kaufmann, S. H. E. (2010) The quest for biomarkers in tuberculosis, Drug Discov. Today 15, 148-157.

19. Zhou, F. B., Xu, X. D., Wu, S. J., Cui, X. B., Fan, L., and Pan, W. Q. (2015) Protein array identification of protein markers for serodiagnosis of Mycobacterium tuberculosis infection, Sci Rep 5, 15349.

20. Pollock, N. R., Macovei, L., Kanunfre, K., Dhiman, R., Restrepo, B. I., Zarate, I., Pino, P. A., Mora-Guzman, F., Fujiwara, R. T., Michel, G., Kashino, S. S., and Campos-Neto, A. (2013) Validation of Mycobacterium tuberculosis Rv1681 protein as a diagnostic marker of active pulmonary tuberculosis, J. Clin. Microbiol. 51, 1367-1373.

21. Napolitano, D. R., Pollock, N., Kashino, S. S., Rodrigues, V., and Campos-Neto, A. (2008) Identification of Mycobacterium tuberculosis ornithine carboamyltransferase in urine as a possible molecular marker of active pulmonary tuberculosis, Clin. Vaccine Immunol. 15, 638-643.

22. Batz, H.-G., Cooke, G. S., and Reid, S. D. (2011) Towards lab free tuberculosis diagnosis, Médecins Sans Frontières Report.

23. Zhao, N., Schmitt, M. A., and Fisk, J. D. (2016) Phage display selection of tight specific binding variants from a hyperthermostable Sso7d scaffold protein library, FEBS J. 283, 1351-1367.

24. Binz, H. K., and Pluckthun, A. (2005) Engineered proteins as specific binding reagents, Curr. Opin. Biotechnol. 16, 459-469.

25. Nygren, P. A., and Skerra, A. (2004) Binding proteins from alternative scaffolds, J. Immunol. Methods 290, 3-28.

26. Edmondson, S. P., and Shriver, J. W. (2001) DNA-binding proteins Sac7d and Sso7d from Sulfolobus, Hyperthermophilic Enzymes, Pt C 334, 129-145.

27. McCrary, B. S., Edmondson, S. P., and Shriver, J. W. (1996) Hyperthermophile protein folding thermodynamics: Differential scanning calorimetry and chemical denaturation of Sac7d, J. Mol. Biol. 264, 784-805.

28. McCrary, B. S., Edmondson, S. P., and Shriver, J. W. (1998) Linkage of protonation and anion binding to the folding of the native Sac7d and Sso7d, Biophys. J. 74, A169-A169.

29. Edmondson, S., and Shriver, J. (1998) Solution structure of the thermophile Sac7d protein and Sac7d-DNA complexes, Biophys. J. 74, A67-A67.

30. Gao, Y. G., Su, S. Y., Robinson, H., Padmanabhan, S., Lim, L., McCrary, B. S., Edmondson, S. P., Shriver, J. W., and Wang, A. H. J. (1998) The crystal structure of the hyperthermophile chromosomal protein Sso7d bound to DNA, Nat. Struct. Biol.5, 782786.

31. Murzin, A. G. (1993) OB(oligonucleotide oligosaccharide binding)-fold - common structural and functional solution for nonhomologous sequences, EMBO J. 12, 861-867.

32. Gera, N., Hussain, M., Wright, R. C., and Rao, B. M. (2011) Highly stable binding proteins derived from the hyperthermophilic Sso7d scaffold, J. Mol. Biol. 409, 601-616.

33. Mouratou, B., Schaeffer, F., Guilvout, I., Tello-Manigne, D., Pugsley, A. P., Alzari, P. M., and Pecorari, F. (2007) Remodeling a DNA-binding protein as a specific in vivo inhibitor of bacterial secretin PulD, Proc. Natl. Acad. Sci. U. S. A. 104, 17983-17988. 
34. Steemson, J. D., Baake, M., Rakonjac, J., Arcus, V. L., and Liddament, M. T. (2014) Tracking molecular recognition at the atomic level with a new protein scaffold based on the OB-fold, PLoS One 9.

35. Shulman, M., Wilde, C. D., and Kohler, G. (1978) Better cell line for making hybridomas secreting specific antibodies, Nature 276, 269-270.

36. Sidhu, S. S., and Weiss, G. A. (2004) Phage display a practical approach, In Practical approach series (Clackson, T., and Lowman, H. B., Eds.), pp 27-41, Oxford University Press, Oxford.

37. Liu, Y. Y., Regula, L. K., Stewart, A., and Lai, J. R. (2011) Synthetic Fab fragments that bind the HIV-1 gp41 heptad repeat regions, Biochem. Biophys. Res. Commun. 413, 611615.

38. Matochko, W. L., Li, S. C., Tang, S. K. Y., and Derda, R. (2014) Prospective identification of parasitic sequences in phage display screens, Nucleic Acids Res. 42, 1784-1798.

39. Menendez, A., and Scott, J. K. (2005) The nature of target-unrelated peptides recovered in the screening of phage-displayed random peptide libraries with antibodies, Anal. Biochem. 336, 145-157.

40. Olofsson, L., Ankarloo, J., Andersson, P. O., and Nicholls, I. A. (2001) Filamentous bacteriophage stability in non-aqueous media, Chem. Biol. 8, 661-671.

41. Weiss, G. A., and Penner, R. M. (2008) The promise of phage display: Customized affinity and specificity, Anal. Chem. 80, 3082-3089.

42. Petrenko, V. A., and Smith, G. P. (2000) Phages from landscape libraries as substitute antibodies, Protein Eng. 13, 589-592.

43. Goldman, E. R., Pazirandeh, M. P., Mauro, J. M., King, K. D., Frey, J. C., and Anderson, G. P. (2000) Phage-displayed peptides as biosensor reagents, J. Mol. Recognit. 13, 382387.

44. Singh, R. K., Tiwari, M. K., Singh, R., and Lee, J.-K. (2013) From protein engineering to immobilization: Promising strategies for the upgrade of industrial enzymes, Int. J. Mol. Sci. 14, 1232-1277.

45. Lang, Q. L., Wang, F., Yin, L., Liu, M. J., Petrenko, V. A., and Liu, A. H. (2014) Specific probe selection from landscape phage display library and its application in enzyme-linked immunosorbent assay of free prostate-specific antigen, Anal. Chem. 86, 2767-2774.

46. Crowther, J. R. (2009) The ELISA guidebook, Humana Press, New York, NY.

47. Rose, C., Parker, A., Jefferson, B., and Cartmell, E. (2015) The characterization of feces and urine: A review of the literature to inform advanced treatment technology, Crit. Rev. Environ. Sci. Technol. 45, 1827-1879.

48. Hicks, C. R. (1993) Fundamental concepts in the design of experiments, Saunders College Pub, New York. 
Table 1. Sso7d library panning details for Mtb OTC.

\begin{tabular}{|c|c|c|c|c|}
\hline Selection Round & $\begin{array}{c}\text { Output (cfu) } \\
\text { against target }\end{array}$ & $\begin{array}{l}\text { Output (cfu) } \\
\text { against } \\
\text { blocked well }\end{array}$ & Enhancement & $\begin{array}{c}\text { ELISA-positive } \\
\text { clones }\end{array}$ \\
\hline 1 & $8 \times 10^{6}$ & N/A & N/A & $\mathrm{N} / \mathrm{A}$ \\
\hline 2 & $5 \times 10^{4}$ & N/A & N/A & N/A \\
\hline 3 & $8 \times 10^{4}$ & $1 \times 10^{4}$ & 8 & N/A \\
\hline 4 & $1 \times 10^{5}$ & $3 \times 10^{3}$ & 33.3 & $7 / 71$ \\
\hline 5 & $7 \times 10^{4}$ & 80 & 875 & $0 / 35$ \\
\hline 3 re-examination & N/A & N/A & N/A & $18 / 24$ \\
\hline $\begin{array}{l}\text { Small colonies were } p \\
\text { colonies showed the } \\
\text { background. }\end{array}$ & $\begin{array}{l}\text { ly chosen off of the } \\
\text { colony morpholog }\end{array}$ & $\begin{array}{l}\text { ound } 3 \text { titer plate } \\
\text { and none exhibit }\end{array}$ & $\begin{array}{l}\text { ELISA examination } \\
\text { ignal greater than th }\end{array}$ & $\begin{array}{l}\text { fter all round } 5 \\
\text { e-fold over }\end{array}$ \\
\hline
\end{tabular}

Table 2. Protein sequences for positions 20-45 of wild type Sso7d and variants selected from the library.

\begin{tabular}{|c|c|}
\hline & $20 \ldots \ldots \ldots 30 \ldots \ldots . \ldots 40 \ldots$ \\
\hline Wild type Sso7d ${ }^{\mathrm{a}}$ & KKVWRVGKMISFTYDEGGGKTGRGAV \\
\hline Sso7d rare arginine template & WSRRRRGKRRRFRYDLGGGKSGIGYV \\
\hline \multicolumn{2}{|l|}{ Target } \\
\hline \multicolumn{2}{|l|}{ Mtb OTC (12 clones sequenced) } \\
\hline Sso7d-MtbOTC-2H12 (2) & WIVRRF GKHISFIYDLGGGKP $G \mathbf{Y} G \mathbf{Y} V$ \\
\hline Sso7d-MtbOTC-5B5 (2) & WPVFRDGKVICFSYDLGGGKRGFGCV \\
\hline Sso7d-MtbOTC-5A6 (2) & WPVYRDGKTICFRYDLGGGKYGYGCV \\
\hline Sso7d-MtbOTC-5B7 (2) & WLVIRHGKNIGFSYDLGGGKYGHGIV \\
\hline Sso7d-MtbOTC-4A8 (1) & WAVGRYGKVIF F RYDLGGGKLGFGYV \\
\hline Sso7d-MtbOTC-5A1 (1) & WAVGRFGKNILFRYDLGGGKYGYGAV \\
\hline Sso7d-MtbOTC-2H8 (1) & WAVARDGKDILFIYDLGGGKFGRGIV \\
\hline Sso7d-MtbOTC-5B4 (1) & WGVGRHGKVIYFYYDLGGGKT GFGAV \\
\hline \multicolumn{2}{|c|}{$\begin{array}{l}\text { a The glutamic acid residue at position } 35 \text { of Sso } 7 \mathrm{~d} \text { was found to contribute to the protein's ribonuclease activity. } \\
\text { The E35L variant lacks ribonuclease activity. The starting template for the phage display library has leucine at } \\
\text { position } 35 \text {. } \\
\text { b The number in parenthesis next to each binding variant denotes the frequency of occurrence of that protein } \\
\text { sequence in the } 12 \text { binding variants characterized. }\end{array}$} \\
\hline
\end{tabular}


Table 3. Fractional factorial experiment setup for analysis of sensor response to simulated urine conditions.

\begin{tabular}{lccr}
\hline $\begin{array}{l}\text { Fractional factorial design } \\
\text { experiment }\left(\mathbf{L 9 ~ 3}^{\mathbf{4 - 2}}\right)\end{array}$ & $\begin{array}{c}\text { Urea } \\
(\mathbf{m g} / \mathbf{L})\end{array}$ & $\begin{array}{c}\text { Ionic strength } \\
(\mathbf{m M})\end{array}$ & $\mathbf{p H}$ \\
\hline 1 & 7000 & 50 & 5.0 \\
2 & 7000 & 165 & 7.4 \\
3 & 7000 & 330 & 10.0 \\
4 & 14000 & 50 & 7.4 \\
5 & 14000 & 165 & 10.0 \\
6 & 14000 & 330 & 5.0 \\
7 & 28000 & 50 & 10.0 \\
8 & 28000 & 165 & 5.0 \\
9 & 28000 & 330 & 7.4 \\
\hline
\end{tabular}




\section{Figure Captions:}

Figure 1. (A) Silver stained polyacrylamide gel of purified recombinant $M t b$ OTC. Lane 1, protein ladder. Lanes 2 and 3, purified recombinant Mtb OTC. $2 \mu$ g of protein were loaded to each lane. (B) Western blot showing Sso7d-MtbOTC binding variant phage particles and the monoclonal antibody mAb-MtbOTC-1B8 binding to $M t b$ OTC. The $M t b$ OTC binding molecule visualized in each lane is indicated along the top of the image.

Figure 2. Sequence and structure of Sso7d from Sulfolobus solfataricus. (A) Primary sequence of Sso7d. Secondary structures are indicated beneath the sequence. The 9 bold, underlined amino acids indicate the diversified positions in the library. The variant used for library construction included an E35L mutation (indicated by *) to abolish Sso7d ribonuclease activity. (B) Sso7d with secondary structural elements color coded to match the cartoon in part A. Side chains shown as sticks indicate positions of diversification. (PDB code: 1BNZ)

Figure 3. ELISA to characterize individual Sso7d-MtbOTC binding variants identified from the phage display library. (A) Cartoon depicting ELISA well set up. (B) Plot of absorbance at 450 $\mathrm{nm}$ vs concentration of phage added per well for eight Ssod7d-MtbOTC binding variants with unique protein sequences. Two-fold dilutions of phage particles were exposed to wells coated with $500 \mathrm{ng}$ of $M t b$ OTC.

Figure 4. Competitive ELISA data for Sso7d-MtbOTC-2H8 phage particles. Phage particles were preincubated with ten different concentrations of soluble Mtb OTC prior to incubation in wells coated with $62.5 \mathrm{ng}$ Mtb OTC. Anti-M13 antibody was used to detect phages that were retained in each well. Measurements were performed in triplicate for each experiment; the $\mathrm{K}_{\mathrm{D}}$ reported represents the average of three independent experiments.

Figure 5. Retention of binding affinity to Mtb OTC by Sso7d-MtbOTC-2H8 phage after exposure to elevated temperatures. ELISA was performed after a 30 minute incubation at temperatures between $50{ }^{\circ} \mathrm{C}$ and $90{ }^{\circ} \mathrm{C}$. ELISA signal for temperature is normalized to the average $\mathrm{A}_{450}$ determined for the sample of Sso7d-MtbOTC-2H8 phage incubated at $0{ }^{\circ} \mathrm{C}$. Each well was prepared by coating with $1 \mu \mathrm{g}$ of $\mathrm{Mtb}$ OTC. Approximately $10^{10}$ particles were added to each well. Bound phage were detected using an anti-M13 antibody.

Figure 6. Sandwich ELISA comparing Sso7d-MtbOTC-2H8 phage particles and mAb-MtbOTC$1 \mathrm{~B} 8$ as capture and detection sensor components. (A) Cartoon depicting sandwich ELISA utilizing the monoclonal antibody mAb-MtbOTC-1B8 as the capture reagent and Sso7dMtbOTC-2H8 phage particles as the detection reagent. (B) Cartoon depicting sandwich ELISA utilizing Sso7d-MtbOTC-2H8 phage particles as the capture reagent and the monoclonal antibody mAb-MtbOTC-1B8 as the detection reagent. (C) ELISA signal for each sandwich ELISA set-up (absorbance at $450 \mathrm{~nm}$ ). Quantities for binding molecules coated to the well represent the amount of each molecule added to each well; binding limits for phage particles and the monoclonal antibody were not evaluated. Binding equivalents for mAb-MtbOTC-1B8 are based on an assumption of 2 binding sites per molecule. Concentrations of the molecule used as the detection reagent were not varied across experiments. 
Figure 7. Sandwich ELISA comparing Sso7d-MtbOTC2-2H8 on phage and off phage as the capture reagent and utilizing the monoclonal antibody mAb-MtbOTC-1B8 as the detection reagent. (A) Cartoon depicting sandwich ELISA utilizing Sso7d-MtbOTC-2H8 phage particles as the capture reagent and the monoclonal antibody mAb-MtbOTC-1B8 as the detection reagent. (B) Cartoon depicting sandwich ELISA utilizing purified Sso7d-MtbOTC-2H8 protein as the capture reagent and the monoclonal antibody mAb-MtbOTC-1B8 as the detection reagent. (C) ELISA signal for each sandwich ELISA set-up (absorbance at $450 \mathrm{~nm}$ ). Quantities for binding molecules coated to the well represent the amount of each molecule added to each well; binding limits for phage particles and purified protein were not evaluated. Binding equivalents for Sso7dMtbOTC-2H8 phage particles are based on an assumption of 1 Sso7d-MtbOTC binding variant displayed per phage particle. Concentrations of the monoclonal antibody mAb-MtbOTC-1B8 used as the detection reagent were not varied across experiments.

Figure 8. Standard curve for the Mtb OTC sandwich ELISA using the monoclonal antiody mAbMtbOTC-1B8 as the capture reagent and Sso7d-MtbOTC-2H8 phage particles as the detection reagent. $11 \mu \mathrm{g}$ of mAb-MtbOTC-1B8 antibody were coated to each well, and $9 \times 10^{11}$ phage particles were added for detection. The absorbance at $450 \mathrm{~nm}$ is plotted as a function of the amount of purified Mtb OTC added to each ELISA well over two orders of magnitude. The inset enlarges the data gathered for the lowest amounts of Mtb OTC.

Figure 9. $M t b$ OTC sandwich ELISA assay performance under various simulated urine conditions. A fractional factorial experiment was designed to evaluate the effects of ionic strength, urea concentration, and $\mathrm{pH}$ on assay performance. Experiments are grouped by the $\mathrm{pH}$ at which the assay was performed. After coating and blocking the wells as described, $10 \mathrm{ng}$ of $M t b$ OTC in each of 10 buffers (9 experimental buffers and standard PBS) were added to the wells. The ionic strength and urea concentration of the buffer utilized for each of the 9 experiments are listed in Table 3. As in the assay format utilized for standard curve evaluation, $11 \mu \mathrm{g}$ of mAb-MtbOTC-1B8 antibody were coated to each well, and $9 \times 10^{11}$ phage particles were added for detection. The absorbance at $450 \mathrm{~nm}$ is plotted relative to the ELISA signal for the assay performed in PBS. 


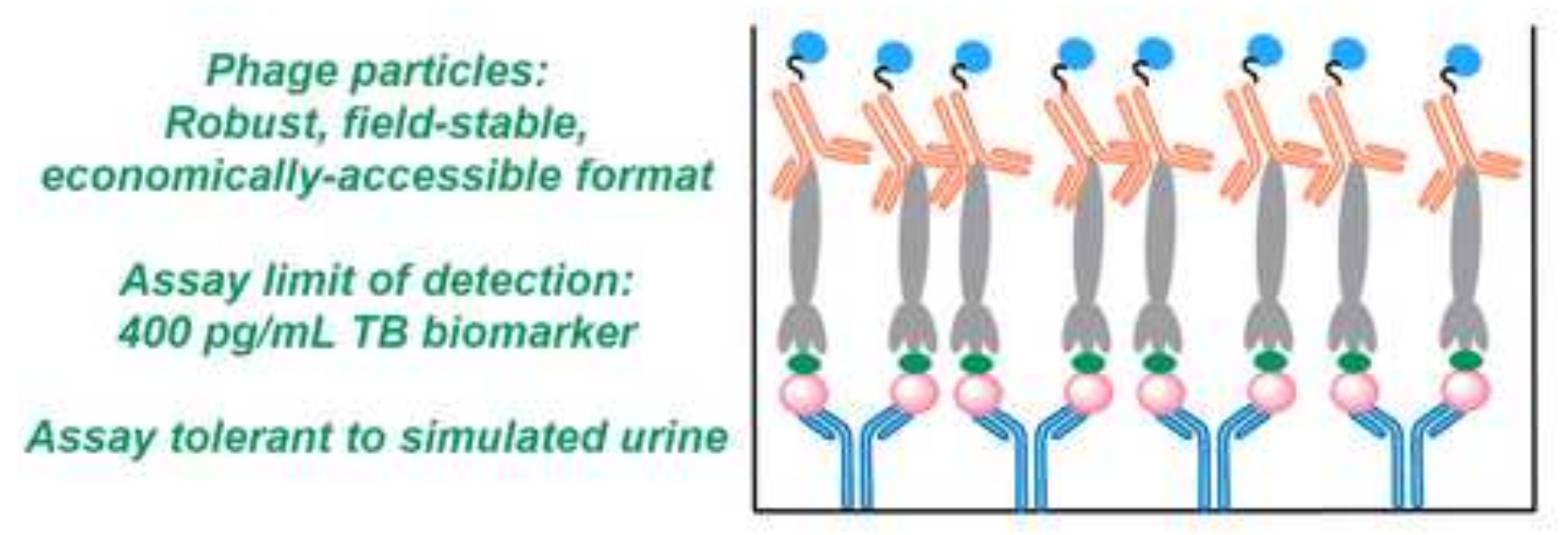

2. Phage particle displaying hyperthermostable binding molecule evolved for TB biomarker

- Monoclonal antibody raised against TB biomarker

Biomarker of active tuberculosis

Anti-phage antibody (signal) 
A

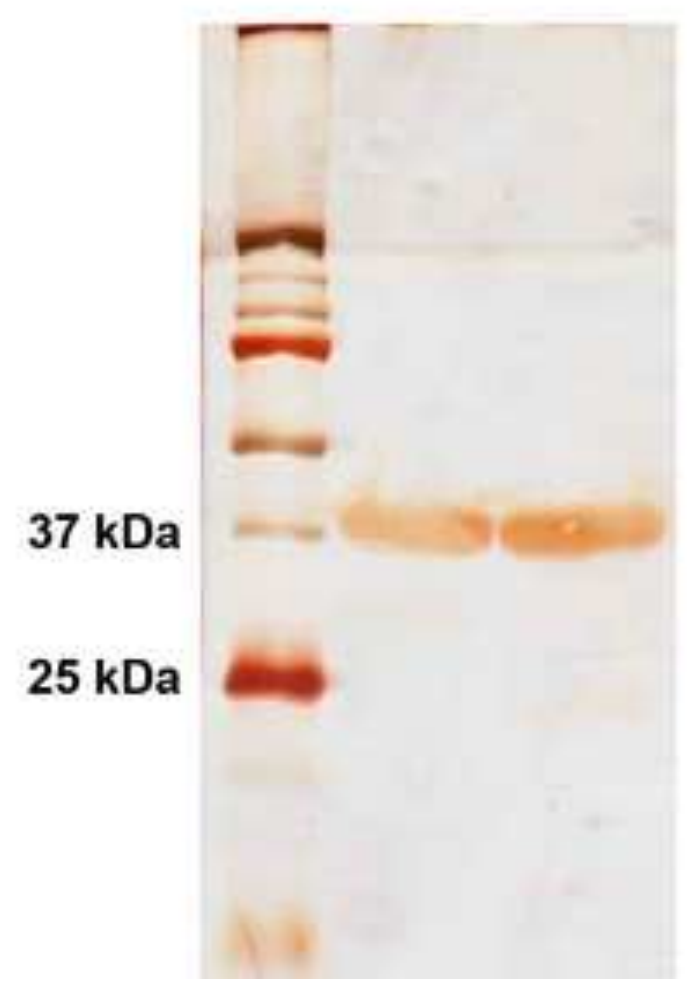

B

Sso7d-MtbOTC binding variant phage

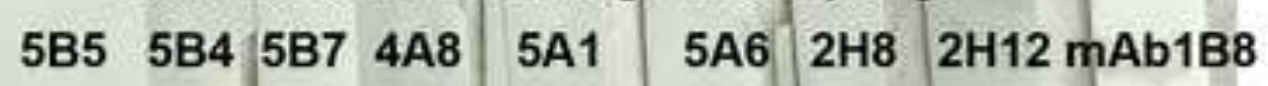

$40 \mathrm{kDa}$

$35 \mathrm{kDa}$

$25 \mathrm{kDa}$

$=$

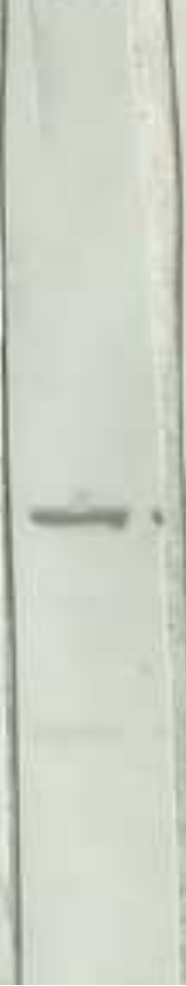


A

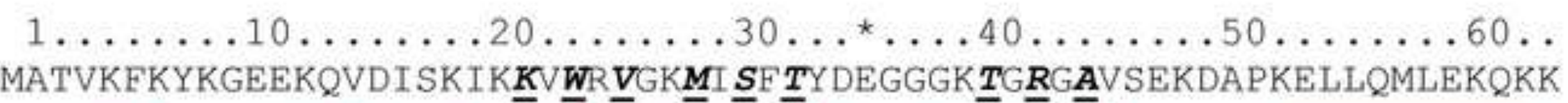
$-$ $\beta 1$

$\beta 2$

$\beta 3$

$\beta 5$

$\alpha 1$

B

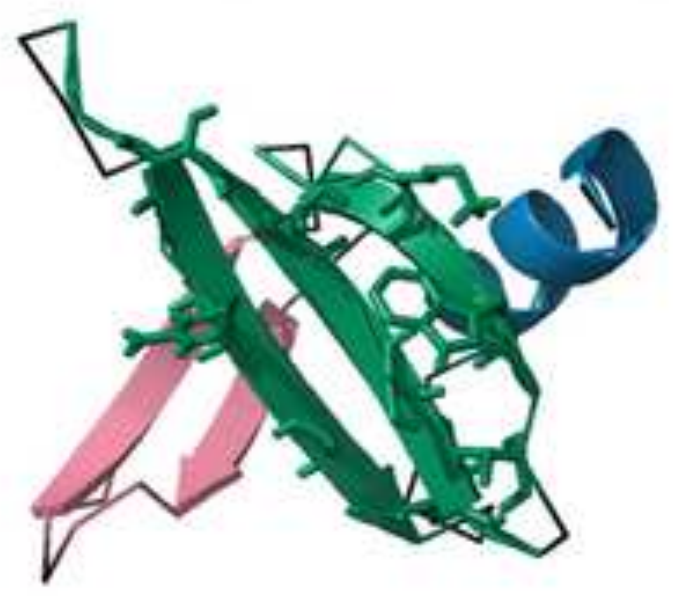


A

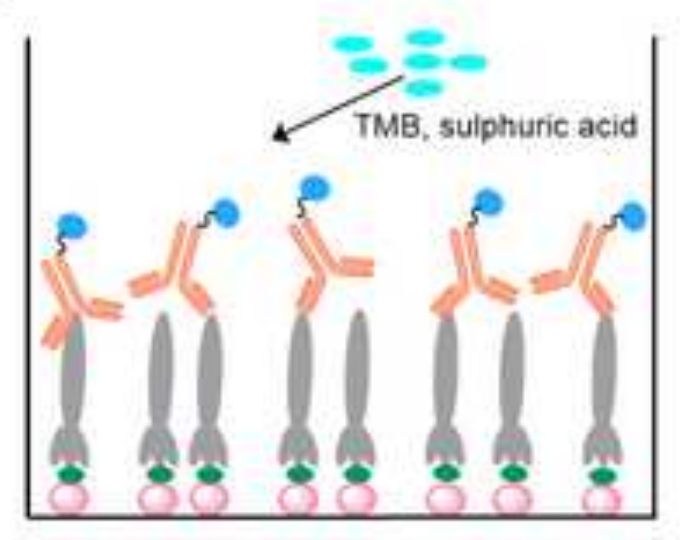

Recombinant Mtb OTC

Phage particle displaying

Sso7d-MtboTC binding variant

anti-M13 ab/HRP
B

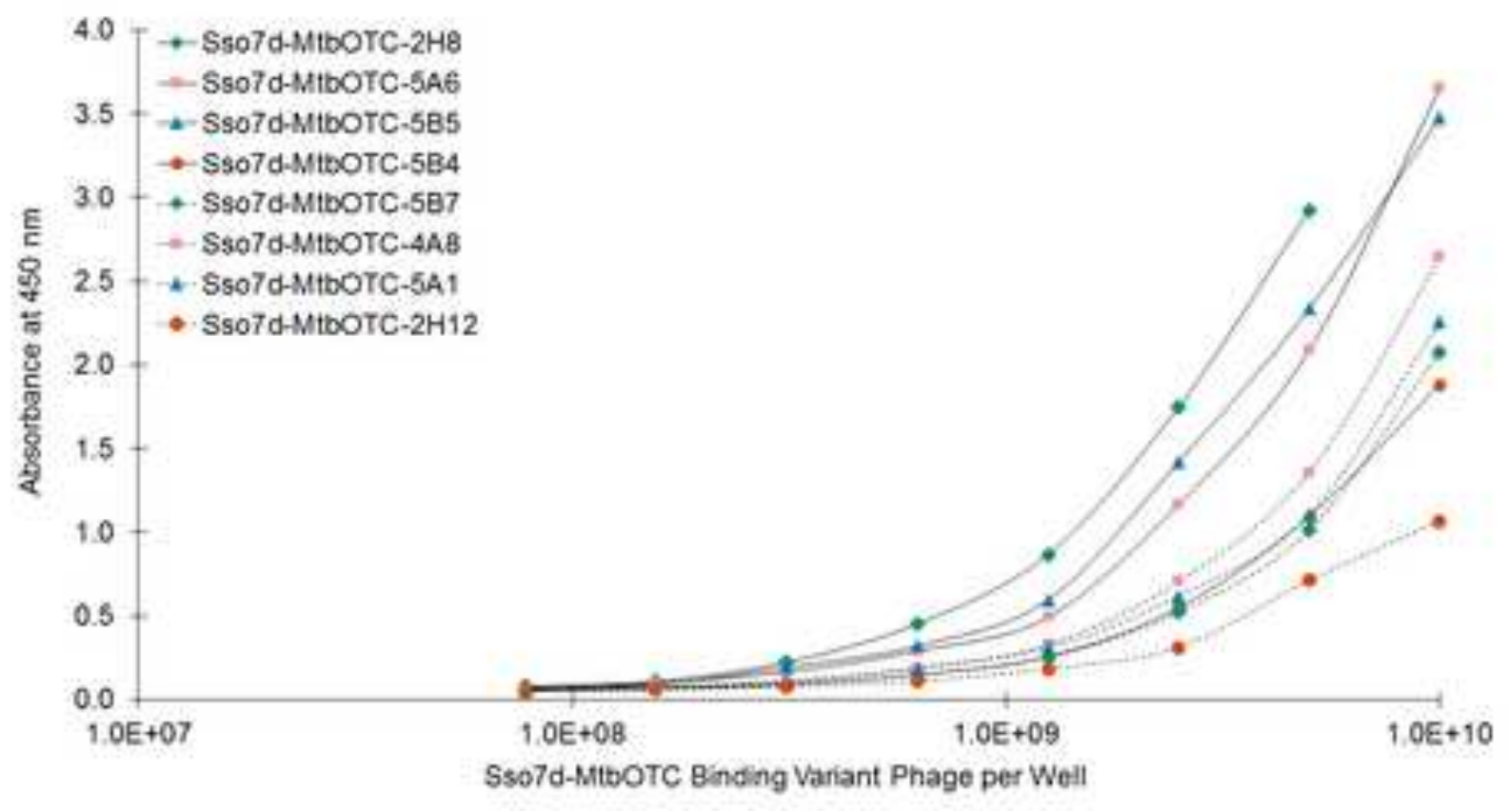




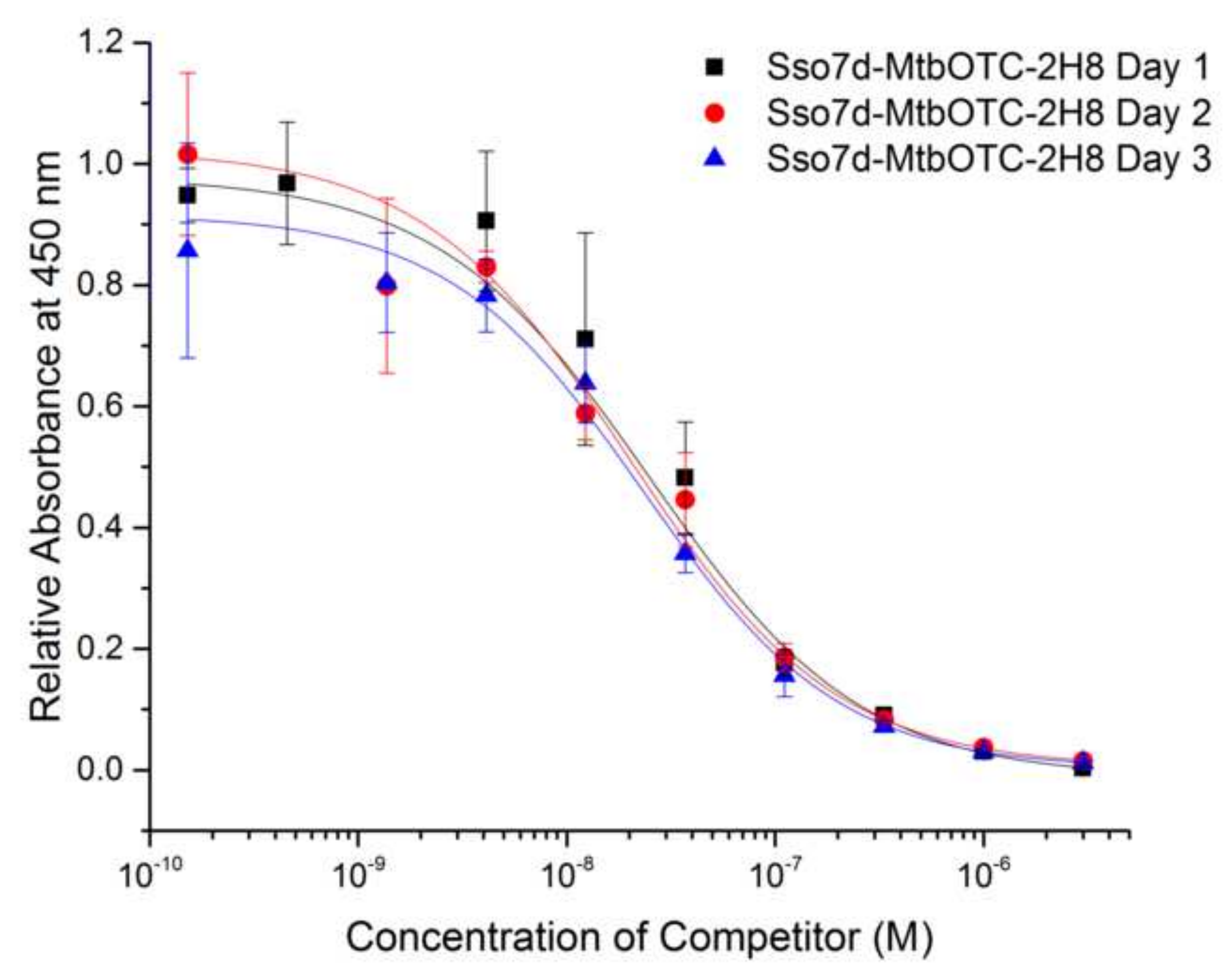




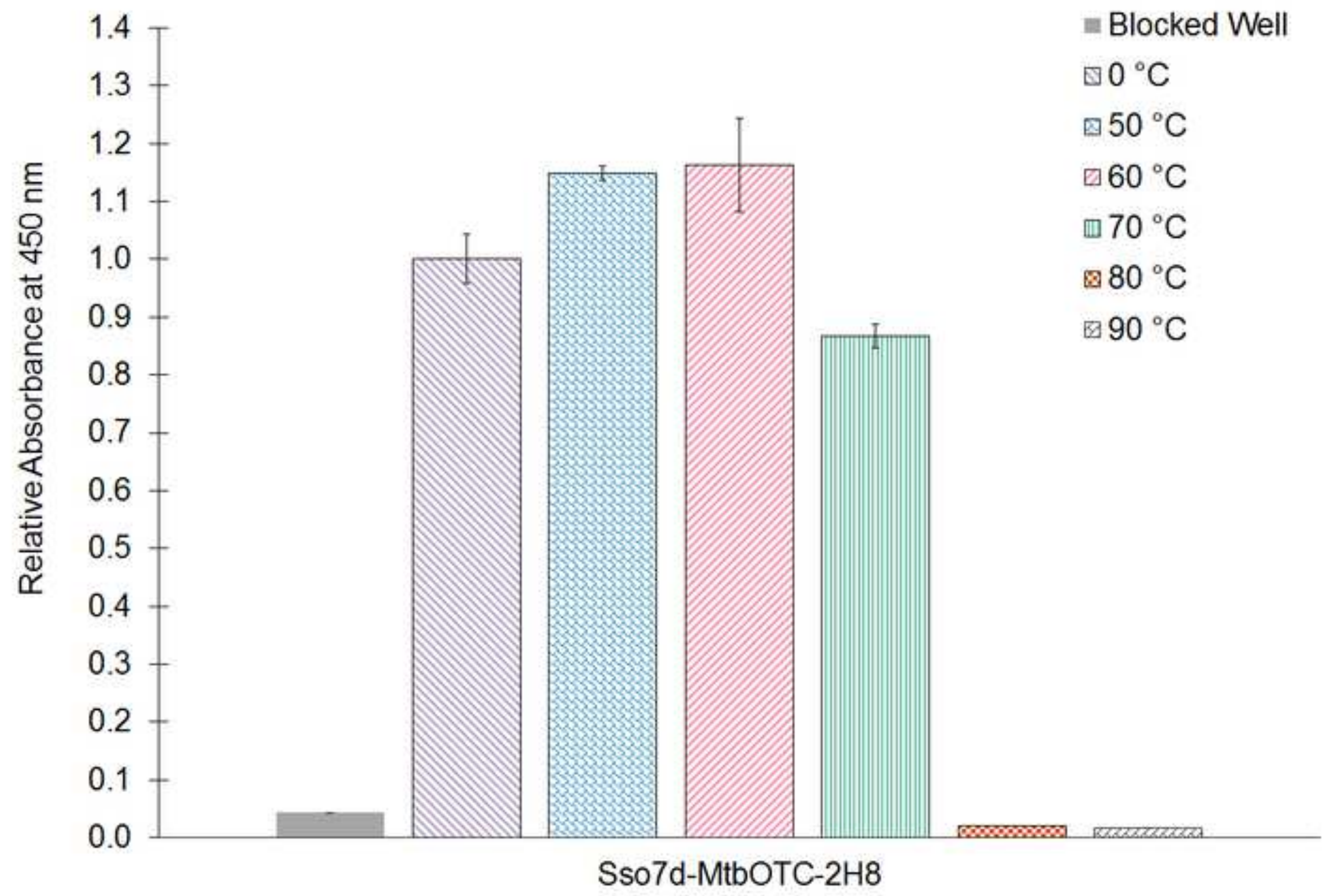


A

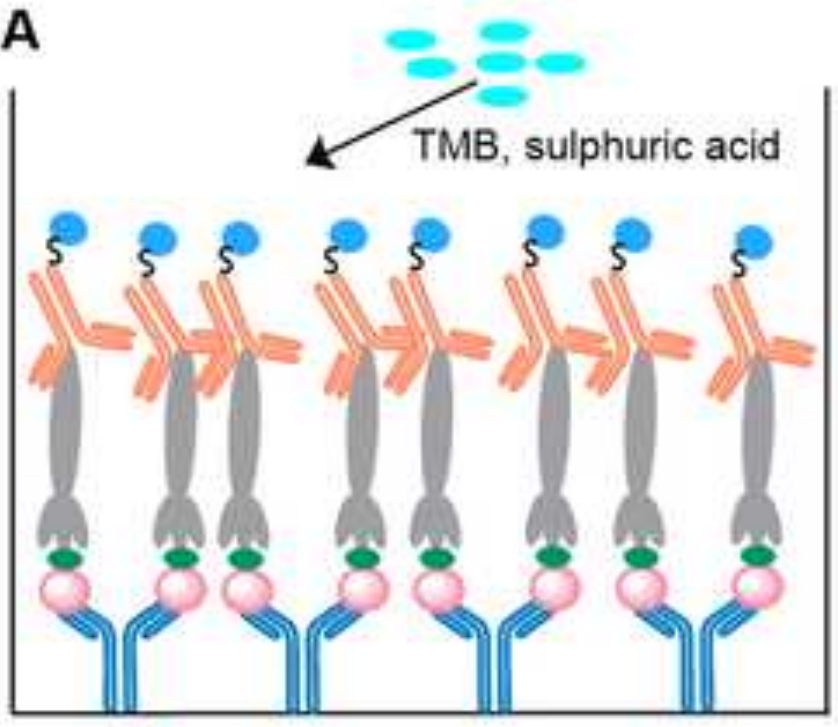

Phage particle displaying

Sso7d-MtbOTC binding variant

7f mab-Mtbotc-1B8

Recombinant Mtb OTC

C

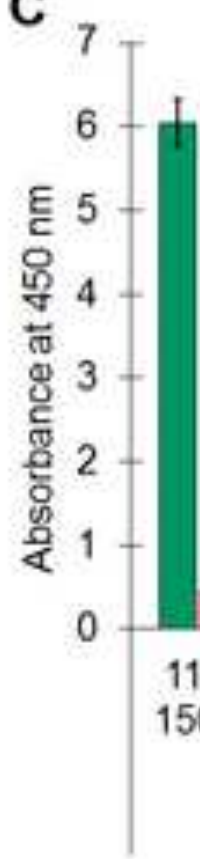

$11.1 \mu \mathrm{g} /$ $150 \mathrm{pmol}$

$1.1 \mu \mathrm{g} /$

$15 \mathrm{pmol}$

$0.1 \mu \mathrm{g} /$

$1.5 \mathrm{pmol}$

mAb-MtbOTC-1B8 coated to wells
B
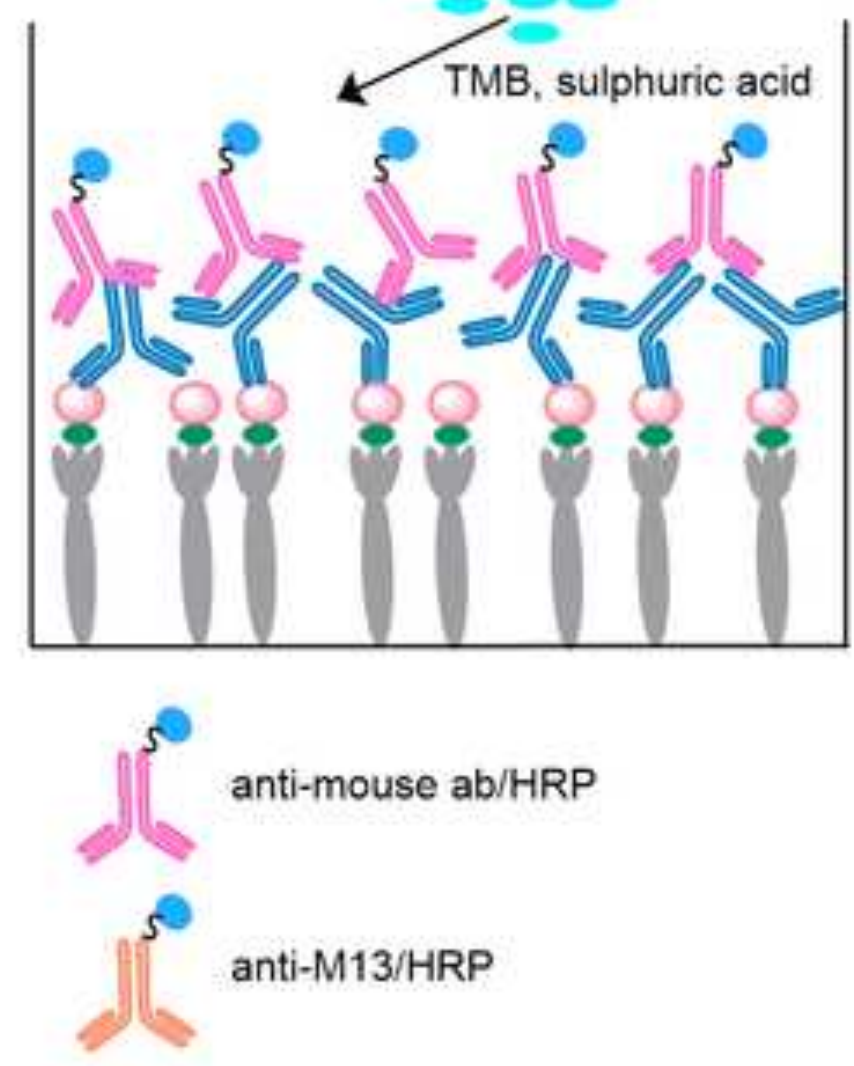

$=\mathrm{Sso} 7 \mathrm{~d}-\mathrm{M}$ tbOTC-2H8 phage for detection, $250 \mathrm{ng}$ Mtb OTC

$\because \mathrm{Sso} 7 \mathrm{~d}-\mathrm{M}$ tboTC-2H8 phage for detection, 0 ng Mtb OTC

= mAb-MtbOTC-1B8 for detection, $250 \mathrm{ng}$ Mtb OTC

= mAb-MtbOTC-1B8 for detection, Ong Mtb OTC

$0.01 \mu \mathrm{g} /$

Sso7d-MtbOTC-2H8 phage coated to wells

Blocked well

Negative 
A

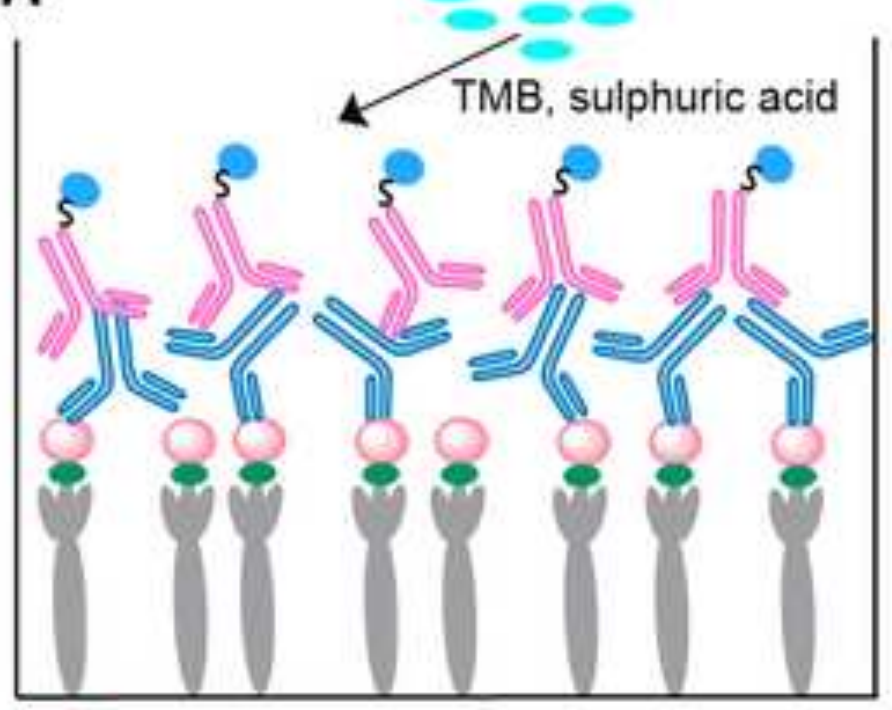

Phage particle displaying

Sso7d-MtbOTC binding variant
B

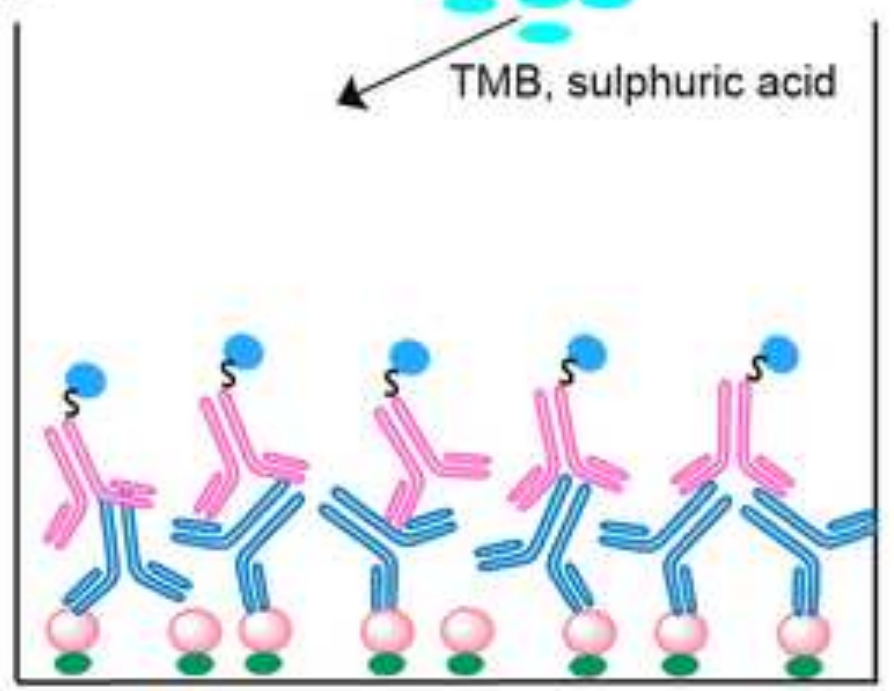

anti-mouse $\mathrm{ab} / \mathrm{HRP}$

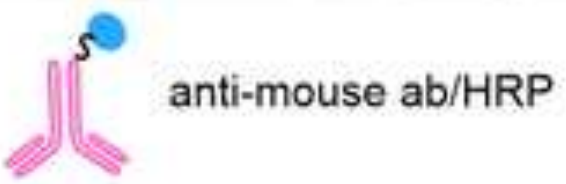

mAb-MtbOTC-1B8

Recombinant Mtb OTC

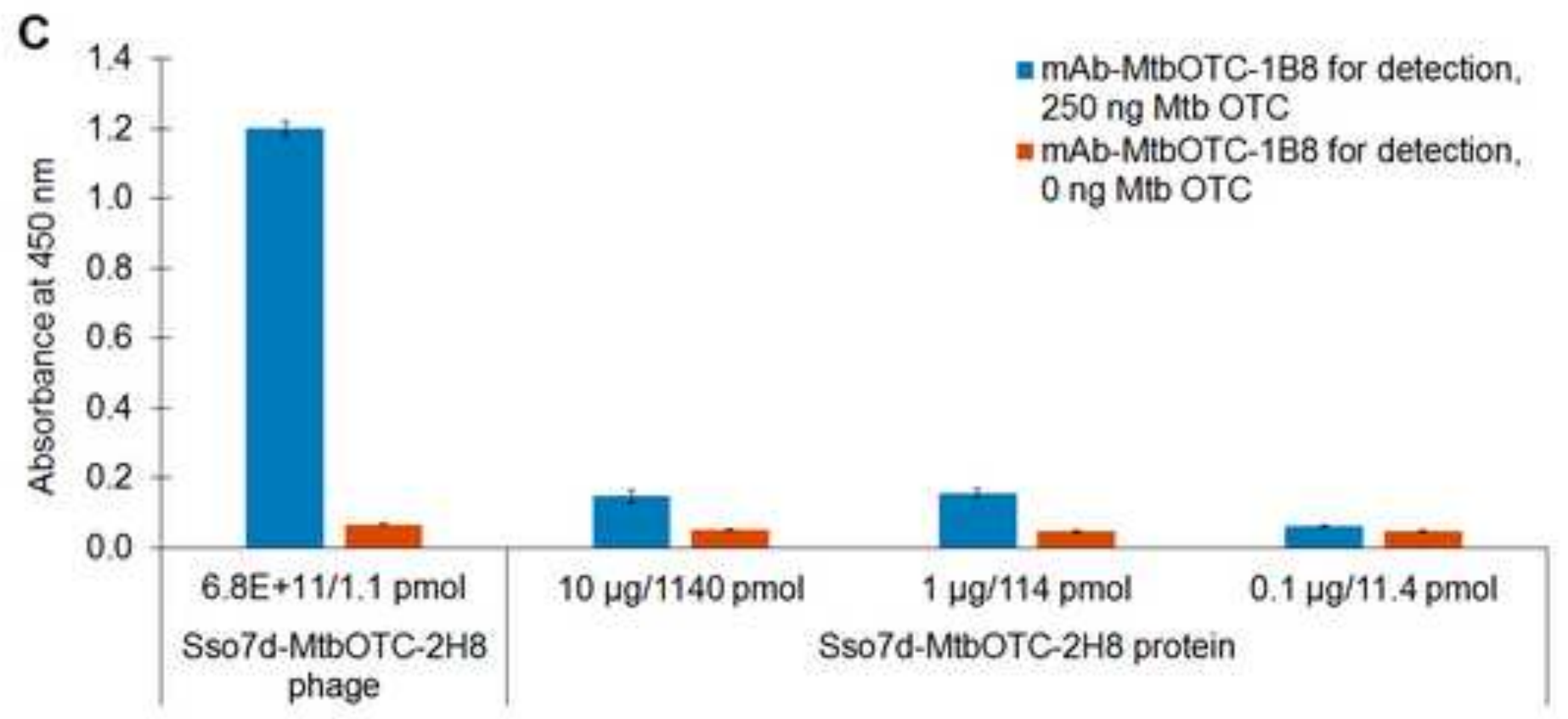




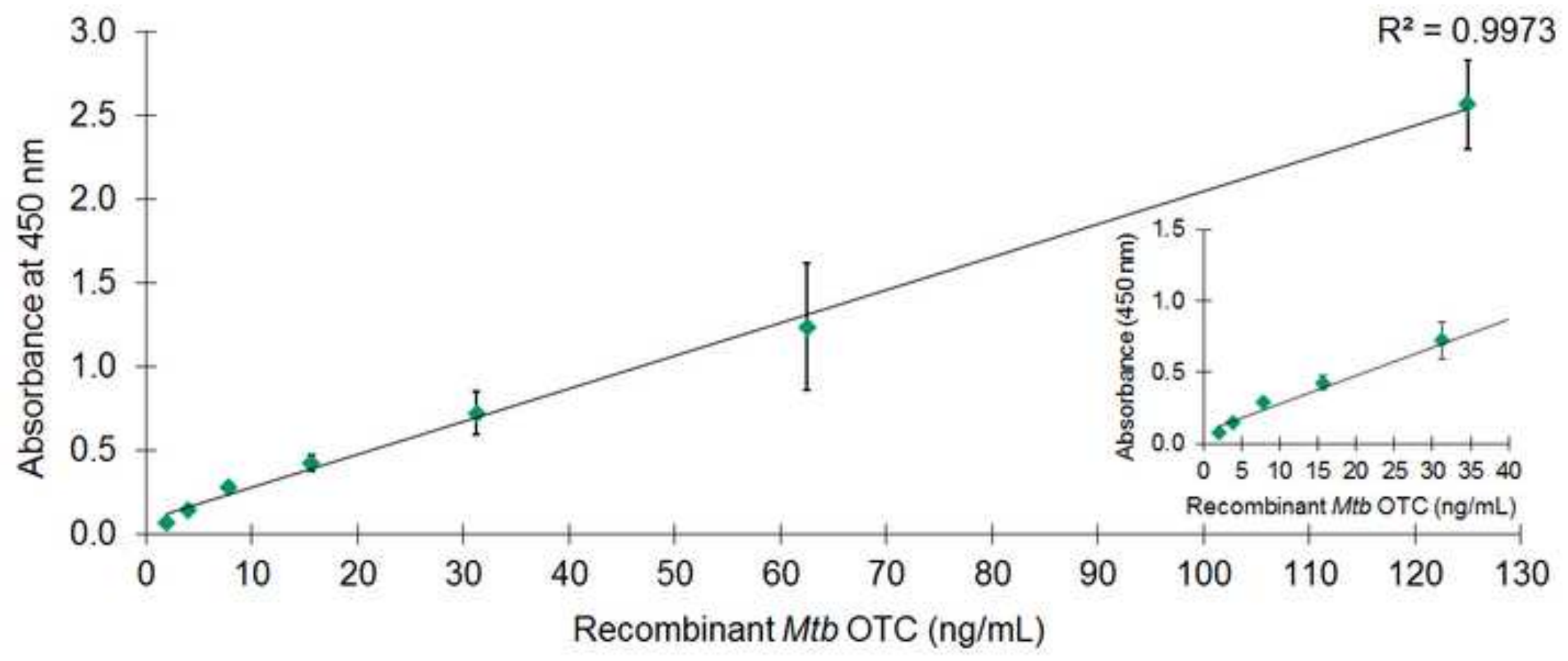




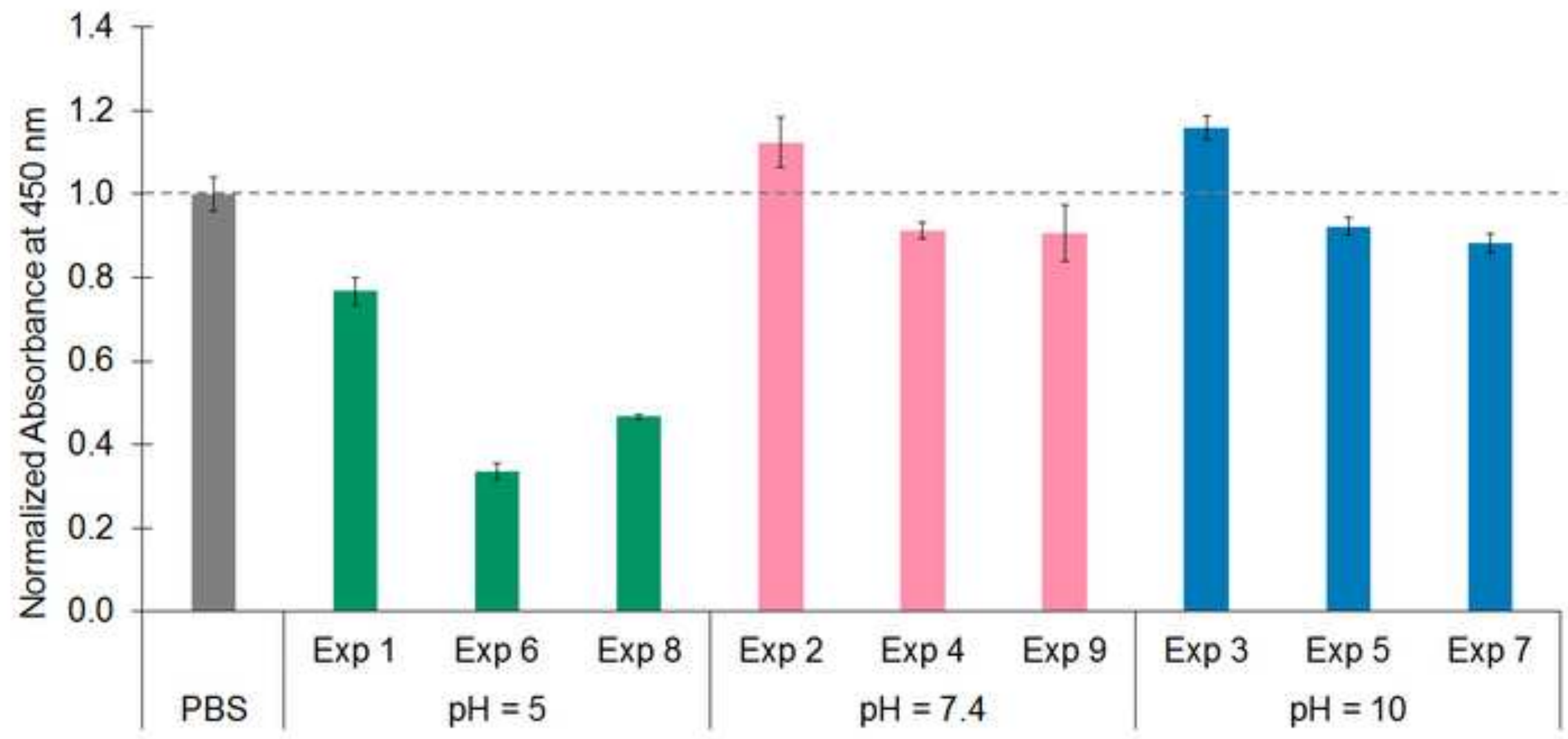

